\title{
Lymphotoxin-alpha expression in the meninges causes lymphoid tissue formation and neurodegeneration
}

Rachel E James Bates ${ }^{1^{*}}, \mathrm{PhD}$, Eleanor Browne ${ }^{1^{*}}, \mathrm{PhD}$, Renee Schalks ${ }^{1}, \mathrm{PhD}$, Heather Jacobs ${ }^{1}$ MSc, Li Tan ${ }^{1}$, MSc, Puja Parekh ${ }^{1}$, MSc, Roberta Magliozzi ${ }^{1,2}, \mathrm{PhD}$, Massimiliano Calabrese ${ }^{2}$, MD PhD, Nicholas D. Mazarakis ${ }^{1}, \mathrm{PhD}$, and +Richard Reynolds ${ }^{1,3}, \mathrm{PhD}$

${ }^{1}$ Division of Neuroscience, Department of Brain Sciences, Imperial College London, Hammersmith, Hospital Campus, UK.

${ }^{2}$ Neurology Section, Dept. of Neurological and Movement Sciences, University of Verona, Italy

${ }^{3}$ Centre for Molecular Neuropathology, Lee Kong Chian School of Medicine, Nanyang Technological University, Singapore.

* Joint first authors.

+Corresponding author: Prof Richard Reynolds, Department of Brain Sciences, Imperial College Faculty of Medicine, Hammersmith Hospital Campus, Burlington Danes Building, Du Cane Road, London, W12 0NN.

Running Title: Lymphotoxin-alpha induced cortical pathology in a model of MS

Key words: Multiple sclerosis, neurodegeneration, demyelination, meninges, inflammation, animal model, 


\title{
Summary
}

Increased release of lymphotoxin-alpha contributes to the pro-inflammatory milieu of the cerebrospinal fluid of MS patients. A persistent elevated expression of this cytokine in the meninges of rats gives rise to chronic inflammation with lymphoid tissue induction and accompanying neurodegenerative and demyelinating pathology in the underlying brain tissue.

\begin{abstract}
Lymphotoxin alpha (LT $\alpha$ ) plays an important role in lymphoid organ development and cellular cytotoxicity in the immune system. LT $\alpha$ expression is increased in the cerebrospinal fluid of naïve and progressive multiple sclerosis (MS) patients and post-mortem meningeal tissue. Here we show that persistently increased levels of LT $\alpha$ in the cerebral meninges can give rise to lymphoid-like structures and underlying MS-like cortical pathology. Stereotaxic injections of recombinant LT $\alpha$ into the rat meninges leads to acute meningeal inflammation and subpial demyelination that resolves after 28 days. Injection of an LT $\alpha$ lentiviral vector induces lymphoid-like immune cell aggregates, maintained over 3 months, including T-cell rich zones containing podoplanin+ fibroblastic reticular stromal cells and B-cell rich zones with a network of follicular dendritic cells, together with expression of lymphoid chemokines and their receptors. Extensive microglial activation, subpial demyelination and marked neuronal loss occurs in the underlying cortical parenchyma. These results show that chronic LT $\alpha$ overexpression is sufficient to induce formation of meningeal lymphoid-like structures and subsequent neurodegeneration.
\end{abstract}

\section{Introduction}

The progressive stages of multiple sclerosis (MS) are characterised by an increasing burden of grey matter pathology that is suggested to play a major role in the irreversible accumulation of sensory, cognitive and motor symptoms (Calabrese et al., 2015a; Fisniku et al., 2008; Harrison et al., 2015; Kutzelnigg et al., 2005; Reynolds et al., 2011). Accumulating 
axon and neuronal loss that are stimulated by an ongoing compartmentalised inflammation are now widely accepted to be one of the main pathological correlates of clinical progression (Calabrese et al., 2015b; Magliozzi et al., 2010; Stadelmann, 2011). Recent studies on human post-mortem tissues have suggested that chronic inflammation in the leptomeninges may be one of the main drivers of cortical pathology (Haider et al., 2016; Howell et al., 2011; Magliozzi et al., 2007; Magliozzi et al., 2018). The presence of leptomeningeal inflammation and the development of tertiary lymphoid-like structures (TLS) in the subarachnoid space (Magliozzi et al., 2007; Serafini et al., 2004) correlate with a gradient of neuronal loss in the cortex that is associated with a shorter time to milestones of clinical progression, including a shorter time to wheelchair use, shorter disease duration and younger age at death (Howell et al., 2011; Magliozzi et al., 2010). These TLS are discrete aggregates of $B$ cells and T cells, plasma cells, antigen-presenting cells and a network of follicular dendritic cells, display varying degrees of organisation and may serve as sites for autoantigen presentation and intrathecal antibody production, both of which are important in driving inflammation in chronic MS (Bell et al., 2019; Magliozzi et al., 2007; Serafini et al., 2004). Meningeal inflammation is seen from the very early stages of MS (Bevan et al., 2018; Lucchinetti et al., 2011) and in multiple CNS locations (Howell et al., 2015; Magliozzi et al., 2010; Reali et al., 2020), but the molecular and cellular mechanisms involved in the formation and maintenance of these immune cell aggregates remains unclear.

The presence of compartmentalised inflammation in the meninges and the underlying gradient of pathology in the cortical GM has given rise to the hypothesis that proinflammatory cytokines and other cytotoxic molecules in the CSF may diffuse into the underlying cortex and directly or indirectly initiate cellular damage (Calabrese et al., 2015b; Dendrou et al., 2015; Gardner et al., 2013; Magliozzi et al., 2007). This also raises the possibility that the same molecule(s) that are involved in stimulating the development of lymphoid tissues could also be responsible for the cortical cell damage. Analysis of the composition of post-mortem and patient CSF of MS cases with increased cortical pathology (Magliozzi et al., 2018; Magliozzi et al., 2020) has revealed the elevated presence of proinflammatory cytokines (eg. IFN $\gamma$, TNF, IL2, IL6), molecules related to B-cell activation (eg. BAFF, APRIL, LIGHT, IL10) and lymphoid neogenesis (eg. LT $\alpha$, CXCL13, CXCL10). Of these 
molecules, lymphotoxin- $\alpha$ (LT $\alpha)$ is known to have a role in cellular cytotoxicity, lymphoid neogenesis and TLS formation (Gommerman and Browning, 2003; Gommerman et al., 2003; Kratz et al., 1996), but its involvement in the pathogenesis of MS has been little studied. Lymphotoxin- $\alpha$ can form a soluble homotrimer that binds to TNF receptors 1 and 2 (TNFR1/TNFR2) and it can also form heterotrimers together with LT $\beta$, as either LT $\alpha 2 \beta 1$ or LT $\alpha 1 \beta 2$, which remain tethered in the cell membrane and bind to the LT $\beta$ receptor (LTRR). The involvement of LT $\alpha$ in the pathogenesis of MS was implicated by its ability to potently induce apoptosis of oligodendrocytes in vitro (Selmaj et al., 1991b), and its expression by CD3+ lymphocytes and microglia at the lesion edge of acute and chronic active MS lesions (Cannella et al., 1997; Raine et al., 1998; Selmaj et al., 1991a). Increased LTa mRNA expression was observed in peripheral blood lymphocytes prior to relapse in RRMS, and expression was strongly upregulated by stimulation with MBP in vitro, suggesting that LT $\alpha$ may play a role in the exacerbation of MS (Matusevicius et al., 1996; Rieckmann et al., 1995). Increased LT $\alpha$ expression has also been demonstrated in peripheral blood B-cells (Romme Christensen et al., 2013) and increased release by CD8+ T-cells in SPMS patients (Buckle et al., 2003).

LT $\alpha$ is known to play a key role in lymphoid tissue organogenesis and maintenance (Gommerman and Browning, 2003). Mice that are deficient in LT $\alpha$ have significant defects in the development of lymphoid organs, specifically lacking secondary lymphoid organs including lymph nodes (LN) and Payer's patches (PP)(De Togni et al., 1994) and are resistant to experimental allergic encephalomyelitis induced in C57BI6 mice by immunisation with MOG peptide 35-55 (Suen et al., 1997). In contrast, ectopic transgenic expression driven by the rat insulin promotor caused chronic inflammation and TLS formation in the kidney and pancreas (Kratz et al., 1996). Whilst LT $\alpha$ has also been shown to be a key player in formation of TLS in the lung during influenza and in synovial tissue in rheumatoid arthritis (Hirose et al., 2018), the role of LT $\alpha$ in chronic leptomeningeal inflammation and CNS TLS formation has yet to be examined.

Meningeal inflammation accompanied by various stages of TLS formation has been reported in several animal models of MS, including the relapsing remitting model of experimental autoimmune encephalomyelitis (rrEAE) in the SJL/J mouse immunised with PLP peptide 
(Magliozzi et al., 2004), chronic progressive EAE in the Biozzi-ABH mouse (Magliozzi et al., 2004), in C57BI/6 mice immunised with an MBP-PLP fusion protein (Kuerten et al., 2012) and following adoptive transfer of MOG-specific Th-17 T-cells into C57BI/6 mice (Mitsdoerffer and Peters, 2016; Peters et al., 2011; Pikor et al., 2015). However, little is known concerning the molecular mediators responsible for initiation and maintenance of such organised immune cell infiltrates and their role in the development of demyelinating and neurodegenerative pathology in the underlying CNS tissues. Our previous studies have demonstrated a close association between the formation of TLS in the subarachnoid space of the cerebral cortex and demyelinating and neurodegenerative pathology in the underlying grey matter, which is not reproduced by any of the EAE models (Howell et al., 2011; Magliozzi et al., 2010; Magliozzi et al., 2019). In order to study this relationship, we have recently developed a novel model in the rat in which the levels of pro-inflammatory cytokines can be chronically and ectopically raised to pathological levels (James et al., 2020). Given the potential role of LT $\alpha$ in both cellular cytotoxicity and TLS formation, here we aimed to show whether acute or chronically elevated levels of LT $\alpha$ in the subarachnoid space of the rat brain can result in meningeal TLS formation and subsequent demyelination and neuronal loss. Establishing the factors that may drive the formation of TLS in MS and their causal relationship to cortical pathology will open up an avenue for the development of an effective approach to slowing the progressive course of MS.

\section{Results}

\section{Expression of LT $\alpha$ in MS CSF and meninges}

A cohort of recently diagnosed drug naïve MS patients were grouped based on the presence of at least 10 cortical lesions (MS-high) or less than 2 lesions (MS-low) from 3D DIR-MRI imaging (cohort described in Magliozzi et al., 2018). Significantly increased levels of LTa were present in the CSF of the MS-high group compared to controls and the MS-low group (Fig. 1A). In a similar way, significantly elevated LTa protein levels (Fig 1B) were found in the CSF of the MS-high post-mortem SPMS brains (>60\% GM demyelination and high levels of immune cell infiltration; Magliozzi et al., 2018), compared to both controls and the MS-low group (<40\% GM demyelination and a low density of diffuse meningeal inflammation). Levels of LTa mRNA in the meninges were also significantly increased in SPMS brains 
compared to controls ( $3.2 \pm 1.2$ fold; $p=0.028$ ) (Fig. 1C). Immunostaining for LT $\alpha$ showed cytoplasmic staining in cells within the meningeal infiltrates (Fig. 1D), many of which costained for the pan T-cell marker CD3 (Fig. 1E-G). Immunohistochemistry for the LTBR showed a diffuse pattern of reticular like staining throughout the meningeal infiltrates (Fig. 1I).

\section{Meningeal inflammation after recombinant cytokine injection in CSF}

The formation of dense cellular infiltrates, down the entire depth of the sulcus and extending across the lateral surface of the cortex was observed between 3-14 days following cytokine injection, with the greatest levels seen between 3-7 days, but were absent in controls that received only a PBS injection (Fig. 2A). Very few immune cells were seen in the underlying cortical parenchyma at any timepoint. The meningeal infiltrate was composed of predominantly CD4+ and CD8+ T cells and CD79a+ B-cells (shown in Fig. 2B at 3dpi). CD4+ and CD8+ T-cells were highest at 3dpi and decreased thereafter until they were almost absent by 14 days (Fig. 2C-D). At both 3 and $7 \mathrm{dpi}$ the numbers of CD4+ cells were approximately twice the numbers of CD8+ T-cells (Fig. 2C-D). Infiltration of CD79a+ B-cells was maximal at 7 days post-injection with numbers staying significantly high at 14 and 21 dpi (Fig. 2E). Whilst CD4+ and CD8+ T-cells were roughly evenly distributed throughout the space with no observable organisation, CD79 $\alpha+B$-cells often formed discrete, dense clusters of cells (Fig. 2F). Approximately 65\% of CD79a+ B-cells co-expressed the proliferation marker $\mathrm{Ki} 67$ at $3 \mathrm{dpi}$ with the percentage of CD79a+Ki67+ cells decreasing over time (Fig. 2G-H).

\section{Demyelination and microglial activation after acute cytokine injection.}

Injection of PBS into the SS of control animals, either naive or those immunised with MOG+IFA or IFA only, did not result in any demyelination or activation of microglia (Fig. 3AC). Rats immunised with low dose rmMOG in IFA that also received a stereotaxic injection of $1 \mu \mathrm{g}$ LT $\alpha$ and $75 n g$ IFN $\gamma$ recombinant proteins into the SS exhibited subpial demyelination of the upper cortical layers and midline layers as early as $3 \mathrm{dpi}$ (Fig. 3D-F), which became even more extensive at 7dpi (Fig. 3G-I) and 14dpi (Fig. 3J-L) and was accompanied by substantial IBA1+ microglial activation. Demyelination extended from the corpus callosum boundary of the sagittal sulcus to the dorsal surface of the cortex and laterally from the midline across the subpial surface of the cortex through to rostral regions. By $21 \mathrm{dpi}$ there was very little apparent loss of MOG staining with only small patches of loss seen mostly in midline regions 
(Fig. 3M), with negligible IBA1+ microglial activation (Fig 3N-O), suggesting that remyelination had occurred. Quantification of the loss of MOG staining demonstrated that demyelination was greatest at $7 \mathrm{dpi}$ when compared to naïve animals with less demyelination at 3 and 14dpi in midline layer I (Fig. 3P) and midline layer II-III (Fig. 3Q). A similar pattern was seen in cortical layers I-IV (Fig. 3R) with significantly less MOG+ myelin staining at $7 \mathrm{dpi}(15.2 \pm 2.25 \%)$ than at $3 \mathrm{dpi}(23.2 \pm 3.9 \%)$ and $14 \mathrm{dpi}(25 \pm 3 \%)$. By $21 \mathrm{dpi}$ there was no quantifiable demyelination in the cortical layers (Fig. 3R), but still some loss of MOG staining in midline regions (Fig. 3P-Q). Quantification of IBA1+ immunofluorescence showed levels peaked at 7dpi in cortical (Fig. 3S) and midline (Fig. 3T) layers, corresponding to the peak in demyelination, but were not significantly different from PBS animals by $21 \mathrm{dpi}$.

\section{Expression of the LT $\alpha$ transgene}

In order to study the chronic effects of increased LT $\alpha$ expression by cells in the meningeal space, we developed a high titre viral vector. Primary meningeal cells could be $100 \%$ transduced with the lentiviral vectors for LT $\alpha$ and GFP, demonstrating a good tropism for the virus (Supplementary Fig 1A-F) with no obvious toxicity (Supplementary Fig. 1G). Transduced meningeal cells continued to produce transgene at increasing levels for several days (Supplementary Fig. 1K-L). Following stereotaxic injection into the subarachnoid space of the sagittal sulcus, as indicated by the presence of monastral blue dye at the injection site, mRNA for the LT $\alpha$ transgene was highly expressed at 90 days after injection in meninges dissected from near the injection site (Fig. 4A). Meningeal cells lining the sagittal sulcus were successfully transduced, as shown by the expression of GFP (Fig. 4B). Strong GFP expression could also be detected in meningeal cells lining the outer surface of the cortex. Immunofluorescence with anti-human LT $\alpha$ antibody demonstrated high levels of expression of the human LT $\alpha$ transgene in transduced meningeal cells down the sagittal sulcus at 28 and 90dpi (fig. 4C). Protein expression from the LT $\alpha$ transgene was detectable in CSF samples and maintained at 28 and 90 days whilst levels were undetectable in GFP vector injected animals (Fig. 4D). Human LT $\alpha$ protein was expressed at high levels in tissue taken from around the injection site, it was also present at low levels in surrounding cortical, but not cerebellum, tissue suggesting some transduction in the cortex, possibly via diffusion down perivascular spaces (Fig. 4E).

\section{Meningeal inflammation after LT $\alpha$ overexpression in the subarachnoid space}


In LT $\alpha$ lentiviral vector injected animals, substantial meningeal infiltrates developed and completely filled the midline subarachnoid space by $28 \mathrm{dpi}$ and were maintained until at least $90 \mathrm{dpi}$ (Fig. 5A), the latest time point studied. No qualitative difference was seen between IFA only and IFA+MOG immunised animals. CD4+ and CD8+ T-cells were roughly evenly distributed throughout the space, whereas CD79a+ B-cells tended to cluster more (Fig. 5B). IBA1+ myeloid cells were also present throughout the aggregates. Quantification of CD4+ and CD8+ T-cell numbers showed no significant difference in numbers between IFA and IFA+MOG animals at both 28 and 90 dpi (Fig. 5C-D), with approximately equal numbers of CD8+ and CD4+ T-cells. Although numbers of T-cells were lower at 90dpi, this still represented substantial infiltration. There was no difference in the number of CD79a+ Bcells between IFA or IFA+MOG animals at 28 or $90 \mathrm{dpi}$ (Fig. 5E) and numbers were similar to both CD4+ and CD8+ T-cells. Similar to the animals injected with recombinant cytokines, after viral vector injection CD79a+ B-cells formed into densely packed focal clusters (Fig. 5F), with immunoglobulin-expressing plasma cells sparsely distributed in the same areas (Fig. 5G). Few or no CD8+ or CD4 T-cells were present within these CD79a+ clusters and a proportion of CD79a+ cells were proliferating at both time points, as demonstrated by coexpression with Ki67 (Fig. 5H). The percentage of Ki67+ B-cells was stable between 28 and 90dpi and was not different between MOG+IFA and IFA only animals, suggesting a consistent level of B-cell proliferation that was not driven by the anti-MOG immune response (Fig. 5I-J). Large aggregates of infiltrating cells were also found along the lateral surface of the cortex (Fig. 5K), composed of CD4+ and CD8+ T-cells (Fig. 5L) and CD79a+ Bcells (Fig. 5M), and were present as T-cell rich regions and B-cell clusters (Fig. 5N).

\section{Tertiary lymphoid structures in the meninges}

The segregation of immune cells in the meninges into T-cell rich regions and large B-cell aggregates with ongoing proliferation was suggestive of TLS formation. Therefore, we further analysed the features of the areas of dense infiltration. A common feature was the presence of many channels of variable diameter, ranging from those the size of large venules (Fig. 6A, green arrows) to smaller sized channels reminiscent of high endothelial venules (HEVs; Fig. 6A, red arrows). Staining for the mucosal addressin cell adhesion molecule (MAdCAM-1) revealed multiple MAdCAM-1+ vessels throughout the length of the sagittal sulcus (Fig. 6B). MAdCAM-1 was expressed by large channels, on cells with the 
morphology of slender squamous endothelium cells (Fig. 6C) and on some process bearing individual cells (Fig. 6D). MAdCam-1 expression by mature stromal organiser cells has been previously reported (Benezech et al., 2010) which this population of cells may represent. Many of the channels were also strongly laminin positive (Fig. 6E), whereas the meningeal blood vessels were only weakly laminin positive. We were unable to further characterise the channels with lymphatic markers as all currently available commercial antibodies for mouse and human failed to react with the rat proteins. A dense matrix of stromal cells called fibroblastic reticular cells (FRC) are commonly found in the T-cell zone of TLS and express the markers podoplanin (gp38) and laminin. Podoplanin was highly expressed throughout the aggregates (Fig. 6F) particularly in regions that contained high numbers of CD4 and CD8+ T-cells. No difference in expression between IFA and MOG+IFA immunised animals was seen, with staining for a dense podoplanin network seen in both (Fig. 6G). No staining was present in naïve or GFP vector injected animals (not shown).

Binding of $L T \alpha$ homotrimers to the TNFR1/2 receptors and $L T \alpha / L T \beta$ heterotrimers to the $L T \beta R$ receptor induces expression of many chemokines involved in organisation of the lymphoid environment, many of which are increased in MS CSF (Magliozzi et al., 2018). To investigate which chemokines resident meningeal cells may produce locally, rat primary meningeal fibroblast cells were treated with $100 \mathrm{ng} / \mathrm{ml}$ of recombinant rat LT $\alpha$. LT $\alpha$ treatment induced significant increases in gene expression for CCL19, CXCL13 and LT $\beta R$ after 24 hours (Fig. 7A). Transcript levels for BAFF, CXCL12 and CCL21 did not change significantly. RT-PCR on tissue dissected from the meninges of LVLT $\alpha$ animals at $28 \mathrm{dpi}$ showed significant increases in the mRNA for CXCR5, CCL19, CCL21, CCR7, CXCR4, BAFF and MadCam compared to GFP injected animals (Fig. 7B). CXCL13 showed the largest increase in mRNA transcript levels (Fig. 7C). Immunostaining for CXCL13 revealed expression throughout the meningeal aggregates (Fig. 7D) that at higher magnification displayed an FDC-like fibroblastic expression pattern with multiple thin processes extending throughout the infiltrates (Fig. 7E). In regions where strong CXCL13 expression was found, a population of follicular dendritic cells (FDC) could be stained for the rat FDC marker ED5 (Fig. 7F). FDCs were typically found in B-cell rich areas, but the more widespread diffuse expression could explain why some CD79a+ cells were found distributed throughout the aggregates, even if in small numbers compared to T-cells (Fig. 5B). As expected, expression of the CXCL13 receptor, 
CXCR5, was found in close association within regions of high CXCL13 staining, along with clusters of CD79a+ B-cells (Fig. 7G).

CXCL12+ expression, which provides a survival signal for incoming T-cells, was present on process bearing cells throughout the meningeal infiltrates (Fig. 7H and I) together with its receptor CXCR4 (Fig. 7J). Co-staining of CCL21 and CXCL12 in T-cell rich regions revealed that they were expressed by distinct populations of cells (Fig. $7 \mathrm{H})$, with no apparent coexpression of the two chemokines. CCL19 and CCL21 also showed differential expression patterns with minimal overlap (Fig. 7K-L). CCL21 could be seen in rounded amoeboid-like cells surrounding large channels (Fig. 7K, white arrows green channel). In contrast, CCL19 was seen in more fibroblastic cells in the areas of infiltration along the edges of the sagittal sulcus (Fig. 7K, white arrow red channel) or cortical surface, in a pattern similar to that seen for CXCL12. Cells expressing the CCR7 receptor for CCL19 and CCL21 were present diffusely throughout the meningeal infiltrates (Fig. 7M).

\section{Demyelination and microglial activation after LT $\alpha$ overexpression}

No observable demyelination was present at 28 or $90 \mathrm{dpi}$ following injection of LT $\alpha$ vector in animals immunised with only IFA (Fig. 8A-B). In contrast, extensive subpial demyelination was found in midline and cortical regions, which reached into deeper cortical layers, at both 28 and 90dpi in LT $\alpha$ vector injected animals immunised with MOG+IFA (Fig. 8A-B). Demyelination in MOG immunised animals was significantly greater at 90dpi than 28dpi in both midline (Fig. 8C; $41.0 \pm 5.2 \%$ of cortical GM myelinated at 28 dpi versus $28.5 \pm 4.6 \%$ at $90 \mathrm{dpi}$ ) and subcortical regions (Fig. 8D; $30.9 \pm 2.0 \%$ myelinated at $28 \mathrm{dpi}$ versus $17.8 \pm 4.0 \%$ at 90dpi). GFP vector injected control animals showed no quantifiable demyelination, regardless of their immunisation status (Fig. 8C-D). Loss of MOG+ myelin extended from layer I directly beneath the pial surface down to layer III (Fig. 8E). In midline regions, loss of myelin extended from the midline into layer III (Fig. 8F). Loss of myelin basic protein (MBP) immunostaining confirmed the presence of demyelination rather than a loss of the MOG protein specifically (Fig. 8G). Microglial activation was extensive and widespread at 90dpi in both IFA only and IFA+MOG immunised LT $\alpha$ vector injected animals when compared to GFP vector injected controls (fig. 8H). In subpial cortical and midline regions, microglia showed a highly activated, ramified morphology, whilst in the deeper cortical layers V and VI directly above the corpus callosum they showed a more amoeboid morphology, (fig. 8I). In midline 
regions microglial numbers in LT $\alpha$ vector injected animals were increased at both 28 and 90 dpi when compared to naive and GFP controls, with no significant difference between MOG+IFA versus IFA only immunised animals (Fig. 8J; $425 \pm 37$ cells $/ \mathrm{mm}^{2}$ in IFA versus $507 \pm$ 74 cells $/ \mathrm{mm}^{2}$ in MOG animals at $\left.90 \mathrm{dpi}\right)$. In cortical layers IBA1+ cells were only significantly increased in MOG-immunised animals (Fig. 8K).

\section{Neuronal loss in cortical layers after persistent LT $\alpha$ overexpression}

In order to determine whether chronic overexpression of LT $\alpha$ for 90 days would lead to cortical neurodegeneration, we counted neuronal numbers using NeuN immunostaining. In midline regions in close proximity to the meningeal aggregates there was a diffuse pattern of loss of neurons in layers I to III that extended the length of the sulcus (Fig. 9A). No neuronal loss was seen in GFP vector injected animals, suggesting this loss was not the result of the surgical procedure. Quantification of midline NeuN+ cell numbers showed a similar loss in both IFA (34.0 $\pm 2.3 \%)$ and MOG (34.0 $\pm 3.3 \%)$ immunised animals compared to both naive and GFP vector injected animals (Fig. 9B). In the cortical parenchyma away from the midline there were expansive regions throughout the motor and sensory cortices that demonstrated a marked decrease in $\mathrm{NeuN}+$ cells, extending from the pial surface into the deeper cortical layers (Fig. 9C). Immunisation with MOG did not impact on neuronal loss (Fig. 9D). In cortical layers II-III there was a $40.0 \pm 2.0 \%$ reduction in neuronal numbers in IFA and $33.0 \pm 3.6 \%$ reduction in MOG immunised animals compared to naive. The cortical region with the most apparent loss of NeuN+ cells was the rostral cortex, where there were focal areas of loss, that in some animals extended from the subpial surface down to layer IV (Fig. 9E). This loss was apparent in all animals injected with LT $\alpha$ vector, both IFA and MOG immunised. We also found a significant reduction in the density of layer $V$ neurons in the midline (Fig. 9F). Whilst there was some loss of larger pyramidal neurons, most of the loss seemed to represent smaller interneurons, although this distinction was not quantified (Fig. 9G). Examination of $200 \mathrm{kDa}$ neurofilament protein immunostaining in midline regions showed a decrease in staining in midline regions accompanying the decrease in neuronal numbers (Fig. 9H).

\section{Discussion}


More extensive cortical demyelination and neurodegeneration in the MS brain, leading to an accelerated disease course (earlier age of clinical onset, more rapid disease progression and earlier age of death), has been shown to be associated with the presence of meningeal TLS formation (Howell et al., 2011; Magliozzi et al., 2007; Magliozzi et al., 2010; Serafini et al., 2004) and an increasingly pro-inflammatory CSF milieu (Magliozzi et al., 2018; 2020). In this study, we provide experimental evidence that chronic production of LT $\alpha$ in the cortical subarachnoid space of the adult rat can induce lymphoid chemokine expression, the persistent presence of meningeal immune cell infiltrates with many features of tertiary lymphoid structures, and chronic pathology in the underlying cortical GM that includes subpial demyelination, microglial activation and accumulating neuronal loss. Such findings are remarkably similar to the pathological changes seen in the MS cerebral cortex in SPMS and provide some possible insights into the pathogenetic mechanisms involved.

Numerous previous studies have shown the presence of LT $\alpha$ and $\beta$ in serum and CSF from progressive MS patients and their production by activated T and B-cells cells (Buckle et al., 2003; Christensen et al., 2013; Corcione et al., 2004; Duddy et al., 2007; Matusevicius et al., 1996). In addition, the gene for LTa (LTA or TNFSF1) has been linked to MS susceptibility (Fernandes Filho et al., 2002) and is suggested to play a role in the stimulation of relapses in RRMS (Navikas et al., 1996; Rieckmann et al., 1995b). However, its role in the link between compartmentalised inflammation and the pathogenesis of CNS damage has received little attention. In our study, LT $\alpha$ post-mortem CSF levels were highest in patients that displayed the greatest levels of meningeal inflammation and the presence of meningeal TLS. Increased levels of LT $\alpha$ gene expression in the isolated meninges strongly suggests a cellular source, most likely from activated memory B-cells or CD4+ and CD8+ T-cells (Duddy et al., 2007; Yang et al., 2018). B-cells from untreated MS patients exhibit abnormal proinflammatory cytokine responses, in particular exaggerated production of LT $\alpha$, when activated in the context of Th1 cytokine IFN? or presence of TLR9 ligand (Bar-Or et al., 2010). Similarly, stimulation with the TLR9 agonist resulted in increased frequency of LT $\alpha$ producing B-cells in patients with RRMS (McWilliam et al., 2018). A significant increase in lymphotoxin secretion from anti-CD3-stimulated CD8+ $T$ cells has also been reported in patients with SPMS compared to normal controls (Buckle et al., 2003), which is in agreement with the immunohistochemical localisation of $\mathrm{LT} \alpha$ in $\mathrm{CD} 3+\mathrm{T}$-cells in the meningeal tissues in the 
current study. Therefore, it is possible that in MS cases with prominent meningeal inflammation, LTa expressed by infiltrating cells within the cerebral sulci may induce a sufficient increase in the local LT $\alpha$ concentration to result in fibroblast differentiation into FDCs and induction of lymphoid chemokines, including CXCL13, CCL19 and CCL21, as is suggested to occur in rheumatoid arthritis synovium (GeurtsvanKessel et al., 2009; Kain and Owens, 2013; Lindhout et al., 1999; van Nierop and de Groot, 2002).

Both acute and chronic elevation of LT $\alpha$ expression in the CSF were able to induce substantial immune cell infiltrates in the meninges that displayed similar cellular composition to the meningeal inflammation reported in SPMS patients (Bell et al., 2019; Magliozzi et al., 2010; Serafini et al., 2004), including those with short duration aggressive disease (Bevan et al., 2018). Although previous animal studies in both active and passive induction EAE models have described the presence of TLS at various stages of complexity in the meninges overlying the brainstem, cerebellum, spinal cord and in the ventricles (Kuerten et al., 2012; Magliozzi et al., 2004; Peters et al., 2011; Pikor et al., 2015), none of these models exhibited the cortical grey matter pathology that is commonly seen in MS. In addition, an association with neurodegeneration in the underlying neural tissue had not been examined. By creating a focal cortical model, with inflammation extending down into the full depth of the sagittal sulcus as well as across the cortical surface, we have reproduced a number of features of the situation seen in MS, notably the presence of TLS neogenesis in deep sulci (Griffiths et al., 2020; Howell et al., 2011). It is likely that the reduced CSF flow in the deep sulcus would allow a local increase in cytokine concentration that might not be reflected by global concentrations, although no experimental data is available on such CSF flow rates in the rat. In this regard it is also of interest that the EAE models in which TLS have been observed were characterised by a more chronic disease course (Kuerten et al., 2012; Magliozzi et al., 2004; Peters et al., 2011; Pikor et al., 2015), which would allow inflammatory infiltrates to build up and be maintained. Whilst TLS developed over a remarkably short time frame in the presence of acutely elevated LT $\alpha$ levels, it required persistent expression for them to be maintained. Persistent ectopic expression of $L T \alpha$ in peripheral organs in transgenic mice has been shown to lead to ectopic lymphoid neogenesis (Drayton et al., 2003; Kratz et al., 1996), but similar experiments have not investigated its role in the CNS and related tissues. The described model allows the 
investigation of some of the consequences of targeted increased LT $\alpha$ expression and subsequent compartmentalised inflammation on tissue damage in a way that is closely relevant to MS itself.

Persistent LT $\alpha$ expression might be expected to create a permissive microenvironment for TLS formation by endowing meningeal stromal support cells 'immune competence' to secrete lymphocyte chemoattractants, such as CXCL13, CCL19 and CCL21, thus producing support networks for the interaction of B-cells with T-cells and local activation and further maturation. Our data suggests that LT $\alpha$ may act on a population of meningeal follicular dendritic cells (FDCS) that then secrete CXCL13 and CCL19 in response to LT $\alpha$ stimulation, allowing the attraction and organisation of B-cells. A similar network of follicular dendriticcells expressing CXCL13 has also been reported in TLS in the MS brain (Magliozzi et al, 2007; Serafini et al., 2004). It has been suggested that these FDCs are of stromal origin and could mature from ubiquitous local precursors such as PDGFRB+ pericytes in response to cytokines such as LT $\alpha$ (Krautler et al., 2012). Discrete patterns of staining also suggest the presence in the meninges of a population of stromal fibroblastic reticular-like cells (FRCS) that express podoplanin, CXCL12 and CCL21 and may help to form T-cell zones. A similar population of podoplanin+ FRCs that secrete CCL21, as well as CXCL13 and BAFF, has been previously reported in the mouse SJL/J EAE model (Pikor et al., 2015).

The presence of many channels of variable diameter within the dense meningeal infiltrates at 28 and 90 days post-LT $\alpha$ vector injection included some that resembled the "loose dilated" lymphatic vessels described previously in TLS in lung biopsies of idiopathic pulmonary arterial hypertension patients (Perros et al., 2012). Smaller channels with thin walls expressing laminin, podoplanin and MadCam-1, with an absence of red blood cells, suggests the formation of high endothelial vessels (HEVs) (Herzog et al., 2013). Unfortunately, expression of LYVE-1 and PNAd could not be used to confirm the presence of true lymphatic vessels as all the human and mouse reactive antibodies failed to react with the rat proteins. While few studies examining TLS in EAE have reported the presence of HEVs in the meninges (Cohen et al., 2021; Dang et al., 2015; Peters et al., 2011), HEVs expressing MadCam-1 and PNad have been reported in MP4-immunised mice (Kuerten et al., 2012). Given that HEVs develop in response to LT $\alpha$ it is possible their formation may only 
occur in the most mature TLOs or in cases with high LT levels (Browning et al., 2005). The meningeal aggregates seen in the current model bear much resemblance to TLS that develop in the kidney and pancreas of rat insulin promoter-LT (RIP-LT $\alpha / \beta)$ mice with regard to cellular composition ( $T$ cells, B cells, plasma cells, and antigen-presenting cells), delineated $\mathrm{T}$ and $\mathrm{B}$ cell areas and characteristic morphologic and antigenic (MAdCAM-1) features of high endothelial venules (Drayton et al., 2003; Kratz et al., 1996). This may indicate a very specific type of TLS that forms in the presence of LT $\alpha$ that may differ from those induced by other cytokines such as IL-17 (Pikor et al., 2015). However, further studies are required to elucidate the detailed cellular and molecular mechanisms by which they form in the cerebral meninges. Although our results show that LT $\alpha$ can play a major role in the initial formation of such structures, other cytokines and chemokines that have been demonstrated to be present in the MS meninges and CSF (Magliozzi et al, 2018) undoubtedly contribute.

Both acute and sustained LT $\alpha$ expression gave rise to subpial demyelinated lesions that extended from the pial surface down to cortical layer III/IV and demonstrated many similarities to key features of MS cortical lesion type III, in particular microglial activation, an absence of amoeboid macrophage-like cells and minimal lymphocytic infiltration. However, subpial demyelination required a pre-existing anti-MOG autoimmunity as very minimal demyelination occurred in IFA only immunised animals. The requirement for an anti-MOG response for demyelination to occur suggests that the microglial activation response may differ depending on prior immunisation, such that activated microglia release different effector molecules in rmMOG-immunised compared to IFA rats, or that autoantibodies contribute to microglial-mediated demyelination in this model. Autoantibodies may also induce demyelination through activation of complement, and immunoglobulin and complement fragment deposition have been observed on myelin sheaths at the lesion edge in targeted EAE following subarachnoid and intracortical injection of TNF and IFN $Y$ (Gardner et al., 2013; Merkler et al., 2006) and in human MS cortical GMLs (Watkins et al., 2016), suggesting that humoral mechanisms may contribute to demyelination in these models and in the current study. In this scenario it is likely that LT $\alpha$ is mainly involved in targeting the immune response to the surface of the cortex, rather than playing a direct cytotoxic role in oligodendrocyte cell death, as has been reported in vitro (Selmaj et al., 1991b). The 
substantial areas of subpial demyelination that were observed in rmMOG-immunised rats at 28 and 90 days post-LVLT $\alpha$, in contrast to the complete remyelination observed by 21 days after recombinant cytokine injection and in other targeted EAE models (Gardner et al., 2013; Merkler et al., 2006; Rodriguez et al., 2014), may represent chronic demyelination due to an eventual failure of remyelination, or overlapping cycles of demyelination and remyelination such that remyelination always appears incomplete. The pattern of demyelination suggests that complete failure ensues after longer time periods, although further studies are required to ascertain this.

Loss of neurons in the grey matter of the cerebral cortex of progressive MS brains, that is independent of demyelination, is a reproducible and widespread finding (Carassiti et al., 2018; Magliozzi et al., 2010; Picon et al., 2021; Trapp et al., 2018; Yates et al., 2017). It is more substantial in cases with higher levels of meningeal inflammation and lymphoid tissue formation, when it occurs in a decreasing gradient from the pial surface towards the white matter (Magliozzi et al., 2010). We report here a similar pattern of neuronal loss in midline and surface cortical regions following chronic expression of LT $\alpha$ in the overlying meninges, which is independent of demyelination or prior MOG immunisation. This result is similar to that obtained previously with TNF+IFN $\gamma$ chronic expression (James et al., 2020), but in the case of LT $\alpha$ did not require the additional presence of IFN $\gamma$. Focal areas of neuronal loss were also found in the rostral cortex, including the somatosensory and secondary somatosensory cortex. Although the dense meningeal infiltrates did not spread this far across the cortex, it is possible that neurons in this region, which have been linked to walking and motor impairment in MS (Arpin et al., 2017a; Arpin et al., 2017b; Strik et al., 2020), are more susceptible to the proinflammatory environment created by overexpression of LT $\alpha$ in the CSF. Further work is needed to understand why these neurons might be particularly vulnerable.

The finding that neuronal loss was similar between MOG and IFA immunised animals and independent of demyelination suggests that LT $\alpha$ can be directly or indirectly cytotoxic to neurons. The LT $\alpha$ homotrimer, unlike the TNF homotrimer, only exists as a soluble protein and can bind to TNFR1, TNFR2 and HVEM receptors (Medvedev et al., 1996). Soluble TNF interaction with TNFR1 is known to predominantly stimulate cell death signaling, either 
apoptosis or necroptosis (Ofengeim and Yuan, 2013; Probert, 2015), whereas binding to TNFR2 is linked with tissue regeneration, including neuroprotection and remyelination (Madsen et al., 2016a; Madsen et al., 2016b; Raphael et al., 2019; Taoufik et al., 2011). Although constitutive expression of TNFR1 is low in the adult brain, it is significantly upregulated in MS and in the cortical GM is expressed predominantly on neurons (Magliozzi et al., 2019b; Picon et al., 2021). In the presence of increased meningeal inflammation and in a pro-inflammatory environment, TNF/TNFR1 mediated signalling in the cortical GM is directed towards necroptotic neuronal death (James et al., 2020; Magliozzi et al., 2019b; Picon et al., 2021). Therefore, it is possible that LT $\alpha$ could also directly stimulate neurodegeneration in the same way by binding to TNFR1, although more in vivo studies would be required to substantiate this. LTa can induce necroptosis with the same potency as TNF in vitro, and like TNF, utilises the RIPK1, RIPK3 and MLKL kinase cascade (Etemadi et al., 2013). The finding that CSF LT $\alpha$ levels were highest in MS patients with the greatest cortical thinning also supports the hypothesis that LT $\alpha$ could be acting directly on neurons following diffusion through the damaged pial membrane (Magliozzi et al., 2010). LT $\alpha$ could also be acting indirectly by stimulating chronic microglial and astroglial activation and subsequent release of neurotoxic mediators (Calabrese et al., 2015b). Increased levels of proinflammatory cytokines in the subarachnoid space have been shown to give rise to increased endogenous expression of TNF and IFN $\gamma$ in the underlying cortical layers (James et al., 2020) and microglia in primary culture release glutamate in response to both LT $\alpha$ and TNF (Gallego-Delgado et al., 2020), raising the possibility that neurodegeneration could occur indirectly via excitotoxicity. However, further experimental studies are required to determine the detailed mechanisms by which LT $\alpha$ could be inducing neurodegeneration in this rat model. Whether stimulation of the formation of TLS in the meninges in this model is due to a direct effect mediated by LT $\alpha /$ TNFR1 interaction or by LT $\alpha$ monomers becoming incorporated into the cell membrane as $L T \alpha_{2} \beta_{1}$ or $L T \alpha_{1} \beta_{2}$ heterotrimers that subsequently bind to the $L T \beta R$, also remains to be determined.

In conclusion, we have demonstrated that overexpression of LT $\alpha$ in the meningeal space in rats leads to chronic cortical pathology that replicates many of the neuropathological and molecular characteristics of MS, including meningeal inflammation with lymphoid tissue formation, microglial activation, demyelination and neuronal loss. We show that chronic 
LT $\alpha$ overexpression alone is sufficient to induce and maintain meningeal TLS. The detailed molecular mechanisms that cause some MS patients to develop meningeal TLS and ways to reduce the formation of these meningeal immune cell infiltrates can now be tested through the use of therapeutic interventions using this experimental model.

\section{Materials and methods}

\section{Human tissue sample selection and immunohistochemistry}

Post-mortem brain tissue was provided by the UK Multiple Sclerosis Tissue Bank at Imperial College London, which obtains tissue through a prospective donor scheme, approved by the National Research Ethics Committee (80/MREC09/31). The diagnosis of all MS cases was confirmed by a consultant neuropathologist according to published guidelines, as described previously (Reynolds et al., 2011). Isolated meningeal tissue samples were dissected from 8 control cases with no neurological disease and 20 secondary progressive MS cases (Magliozzi et al., 2018). Details of each case are listed in Supplementary Table 1. Cases were classed as PM-MS high or PM-MSlow based on numbers of haematoxylin staining cell nuclei in 2 meningeal infiltrates and presence and extent of subpial grey matter demyelination, as described previously (Magliozzi et al., 2018). Protein levels of LT $\alpha$ were determined in postmortem CSF obtained at autopsy from 20 SPMS, 10 with high (PM-MShigh) and 10 with low (PM-MSlow) meningeal inflammation, and 10 healthy controls, using immune-assay Luminex technology (Bio-Plex X200 System equipped with a magnetic workstation, BioRad, Hercules, CA, USA). All samples were run undiluted, in duplicate in the same experiment and in two consecutive experiments, in order to verify the reproducibility and consistency of the results as previously described (Magliozzi et al., 2018).

Naïve relapsing remitting MS patients were screened at diagnosis by 3T-MRI and stratified into MSHIgh $(n=18)$ and MSLow $(n=13)$ by the presence of at least 10 cortical lesions or less than 2 cortical lesions respectively (Supplementary table 2), as described for this patient cohort previously (Magliozzi et al., 2018). The control cohort $(n=10)$ consisted of subjects with non-inflammatory neurological disease. CSF samples from patients were acquired at least 2 months from the last relapse, according to the Consensus guidelines for CSF and Blood biobanking. Samples were centrifugated before storage of the supernatant at $-80^{\circ} \mathrm{C}$ 
until use. Levels of LT $\alpha$ were assessed using immune-assay multiplex techniques on the Luminex technology (Bio-Plex X200 system equipped with a magnetic workstation, BioRad, Hercules, CA), in the same way as the post-mortem samples.

\section{Quantitative reverse transcription polymerase chain reaction}

Meningeal tissue was isolated by manual dissection using a scalpel from up to 16 snapfrozen cortical blocks per MS and control case to give a total mass of 100-250mg tissue per case (Gardner et al., 2013). RNA was extracted using an RNeasy Lipid Tissue Mini Kit (Qiagen) and quantified by Nanodrop ND1000 spectrophotometer. Reverse transcription of $1 \mu \mathrm{g}$ RNA per case to complimentary (CDNA) was performed using a Quantitect Reverse Transcription Kit (Qiagen) with integrated genomic DNA removal. Prime Time qPCR Assays (Integrated DNA Technologies, Iowa, USA) were used to quantify expression of LTa relative to the expression of two housekeeping genes (HKG; glyceraldehydes-3-phosphate dehydrogenase, GAPDH and Xpnpep1). Xpnpep1 is a stable HKG in post-mortem CNS tissue, with no known associations with neuroinflammatory or degenerative pathologies (Durrenberger et al., 2012). Reactions of $20 \mu \mathrm{l}$ were performed in triplicate for each case on a Stratagene MXP4000 system (Agilent Technologies), with MxPro 2007 software. An interplate calibrator did not detect any significant inter-plate differences in efficiency between successive runs. The thermal profile was $95^{\circ} \mathrm{C}$ for $10 \mathrm{~min}, 50$ cycles with a two-step program $\left(95^{\circ} \mathrm{C}\right.$ for $15 \mathrm{~s}, 60^{\circ} \mathrm{C}$ for $\left.45 \mathrm{~s}\right)$. Changes in genes expression were calculated using $\Delta \Delta \mathrm{Ct}$ method.

\section{Lentiviral vector production}

Lentiviral (LV) vectors expressing human LT $\alpha$ (LVLT $\alpha)$ or enhanced green fluorescent protein (LVGFP) were produced as described previously (James et al., 2020). We utilised a HIV-1 transfer plasmid (326-pRRL-sincppt-CMV-eGFP-WPRE genome plasmid) that carries the human cytomegalovirus promoter (CMV) for lentiviral production. Complementary DNA sequences for human lymphotoxin alpha (LT $\alpha)$ were codon optimised for rat including a $5^{\prime}$ Kozak sequence and synthesised by Gene Art with Xba1 and Sal1 restriction sites (Life Sciences, Paisley, UK). The human transgene DNA fragments were excised by restriction digest with Xba1 and Sal1 before purification using preparative agarose gel electrophoresis. The eGFP was removed from the transfer plasmid by digestion with Xba1 and Sal1 and the transgene DNA fragments ligated in-frame using the same restriction sites. 
Recombinant HIV-1 based lentiviruses were produced using four plasmid co-transfection of HEK-293T cells as described previously (James et al., 2020). Briefly, HEK-293T cells were transfected with $15 \mu \mathrm{g}$ vector plasmid (pRRLsincppt-CMV-LT $\alpha-W P R E$ or pRRLsincppt-CMVGFP-WPRE), $15 \mu \mathrm{g}$ of the packaging vector plasmids expressing the HIV-1 gag/pol gene

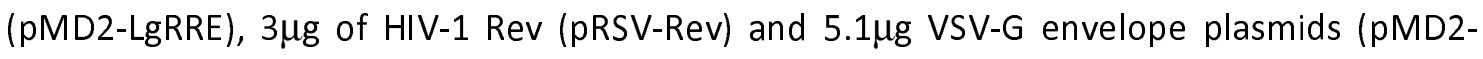
VSV-G) following the addition of $2 \mathrm{~mol} / \mathrm{L} \mathrm{CaCl}$. Lentivirus was concentrated from supernatant using ultracentrifugation and the genome copy number was calculated using the Clontech Lenti-X qRT-PCR Titration kit (Takara).

\section{Transduction of HEK cells}

Hek-293T cells, obtained from ATCC, were maintained at 37C, 5\% CO2 environment and cultured in Dulbecco's modified Eagle's medium (Sigma, UK), supplemented with 10\% Newborn Calf serum (Heat inactivated, Sigma UK), $2 \mathrm{mM}$ penicillin/streptomycin and $2 \mathrm{mM}$ L-glutamine (Sigma, UK). Hek-293T cells were plated in 24 well plates for 24 hours before transduction with LV-LT $\alpha$ or LV-GFP at MOI 5, 10, 20, 35 and 50 and the supernatant harvested after 24 and 48 hours.

\section{Transduction of primary meningeal cultures}

Primary meningeal cultures were prepared from P4 Sprague Dawley pups. Briefly, the meninges were dissected from the surface of the cortex and digested with papain and DNAse. Cells were plated on poly-l-lysine coated 8-well chamber slides or 24 well plates (Corning) and grown in DMEM/F12 (Sigma, UK) supplemented with 10\% calf serum, 1\% Penicillin/Streptomycin and 1\% L-Glutamine (Sigma, UK). After 1 week in culture cells were transduced at various MOls with LV-LT $\alpha$, or LV-GFP and the supernatant harvested after 72 and 96 hours and 7 days. Enzyme-linked immunosorbent assay for human LT $\alpha$ was performed in triplicate using the DUO set ELISA kit (DY210, R\&D Systems, Abingdon, UK). Protein was extracted from transfected meningeal cells using RIPA buffer (R0278, Sigma) and run on Western blot at 20ug per well. Protein was stained with an antibody specific for human LT $\alpha$ to confirm expression.

For gene expression analysis of meningeal cells, $1 \times 10^{5}$ cells were plated in 6 wells plates and treated with 100ng recombinant human LT $\alpha$. RNA was extracted using TRIzol reagent according to manufacturers instructions and $1 \mu \mathrm{l}$ of RNA converted to cDNA using High- 
capacity cDNA reverse transcription kit (Thermofisher, Uk). Prime Time qPCR Assays (Integrated DNA Technologies, Iowa, USA) were used to quantify expression of LTa relative

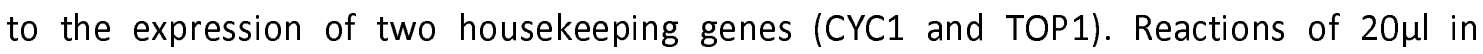
PowerUp SYBR green (Thermofisher, UK) were performed in triplicate for each case on a Stratagene MXP4000 system (Agilent Technologies), with MxPro 2007 software. The thermal profile was $95^{\circ} \mathrm{C}$ for $10 \mathrm{~min}, 40$ cycles with a two-step program $\left(95^{\circ} \mathrm{C}\right.$ for $5 \mathrm{~s}, 60^{\circ} \mathrm{C}$ for 30s). Changes in genes expression were calculated using $\Delta \Delta \mathrm{Ct}$ method.

\section{Immunofluorescence of primary meningeal cells}

For immunofluorescence of transfected meningeal cells grown in 8-well chamber slides, cells were fixed with cold 4\% PFA for 1 hour at room temperature. After fixation, cells were incubated with $0.1 \mathrm{M}$ PBS- $0.1 \%$ triton-X-100 for 1 hour. Primary antibodies were added at their corresponding working concentration for 1 hour at room temperature. After primary antibody incubation, cells were thoroughly rinsed in 0.1M PBS, three times 10 min each. After rinsing cells with incubated with appropriate secondary antibody conjugated to the appropriate fluorophore for 1 hours at room temperature. The cells were rinsed in 0.1M PBS (three times, $10 \mathrm{~min}$ each) and $\mathrm{dH}_{2} \mathrm{O}$, nuclear counterstained with DAPI (diluted in 1:10,000). Lastly, the tissues were mounted with Vectashield Antifade Mountant and coverslipped (24x50mm, VWR, International).

\section{Animals}

All in vivo procedures were performed under UK Home Office approval. Eight to ten week old female Dark Agouti (DA) rats (140-160g) were obtained from Janvier (France) and kept in groups of four in a $12 \mathrm{~h}$ light/dark cycle with food and water provided ad libitum.

\section{Induction of sub-clinical experimental autoimmune encephalomyelitis}

Sub-clinical MOG-induced EAE was induced as described previously (Gardner et al., 2013; James et al, 2020). Rats were anaesthetised with isofluorane and immunised intradermally at the base of the tail (dorsal aspect) with $5 \mu \mathrm{g}$ of recombinant mouse MOG (amino acids 1119 , corresponding to the external Ig-like domain) diluted in sterile PBS and emulsified in an equal volume of incomplete Freund's adjuvant (IFA, Sigma; total volume 100 $\mu$ l per rat). IFA control rats were immunised with PBS emulsified in an equal volume of IFA, and naïve agematched control rats $(n=6)$ received no treatment. For all groups $n$ was 5 to 7 animals with a total of 92 animals used for all experiments. All animals were weighed, examined and 
clinically scored daily according to a scoring system to define the level of neurological deficit, as described previously (Papadopoulos et al., 2006). This dose of recombinant MOG protein has been previously shown to elicit only sub-clinical EAE in DA rats of similar age and weight (Gardner et al., 2013), with production of peripheral anti MOG IgG1 and IgG2a antibody titres previously confirmed to be present in MOG immunised but not IFA immunised animals (James et al., 2020).

\section{Subarachnoid injection of cytokines and LV vectors}

Following immunisation, stereotaxic injections of recombinant cytokines or lentiviral (LV) vectors into the subarachnoid space (SS) were performed under isofluorane anaesthesia at 20-23 days post immunisation, or at an equivalent time-point in age-matched naive control animals. A $5 \mathrm{~mm}$ hole was drilled in the skull at $0.9 \mathrm{~mm}$ caudal to bregma at the level of motor cortex. A finely calibrated pulled glass capillary needle attached to a 265 fixed needle Hamilton syringe $(10 \mu \mathrm{l})$ was lowered to a depth of $2.3 \mathrm{~mm}$ ventral to the dural membrane. The cytokines or lentiviral vectors were infused at a rate of $0.2 \mu \mathrm{l} / \mathrm{min}$ over a period of 20 minutes. Monastral blue (copper II phthalocyanine-tetrasulfonic acid tetrasodium salt; Sigma-Aldrich) was added as a tracer to determine the location of the injections, and resulted in no pathology, as confirmed by previous studies (Kerschensteiner et al., 2004; Merkler et al., 2006; Rodriguez et al., 2014). For lentiviral injections, $4 \mu$ l of lentiviral vector solution diluted in TSSM with $0.5 \mathrm{mM}$ monastral blue at a total of $5 \times 10^{8}$ genomic copies (GC) for LT $\alpha$ and GFP was injected at a rate of $0.2 \mu \mathrm{l} / \mathrm{min}$. For recombinant cytokine injections, control animals received an injection of sterile PBS $0.5 \%$ BSA $(n=5)$. Recombinant human lymphotoxin-alpha ( $h L T \alpha$, reconstituted in $0.1 \%$ (w/v) BSA in sterile PBS; Peprotech) and recombinant rat IFN $\gamma$ (reconstituted in $\mathrm{pH} 8$ sodium phosphate [10mM; Sigma-Aldrich] were diluted to the required concentrations (LT $\alpha 1 \mu \mathrm{g}+$ IFN $\gamma 75 \mathrm{ng}$ per injection) and injected at a total volume $2 \mu \mathrm{l}$ per rat. For all injections, the glass capillary needle was left in place for 10 min after the injection to allow diffusion of the injected solution away from the injection site before slow retraction of the capillary. A group of age-matched naïve rats, which received no immunisation or SS injections, provided an additional control $(n=6)$.

\section{Tissue processing and CSF analysis}

At the termination of the experiment rats were killed by intraperitoneal injection of 200 $\mathrm{mg} / \mathrm{kg}$ of sodium pentobarbitone and a $100 \mu \mathrm{l}$ CSF sample taken from the cisterna magna 
using a $26 \mathrm{G}$ needle (Hamilton) before cessation of breathing, with the animal on a stereotaxic frame. Animals were perfused with $100 \mathrm{ml}$ of PBS followed by $400 \mathrm{ml}$ of $4 \%$ PFA through the left ventricle at 3, 7, 14, 21, 28 and 56 days post viral injection. Brains were removed and post fixed in 4 \% PFA (4 hrs room temp), prior to cryoprotection in $30 \%$ sucrose solution (w/v in PBS) for 72 hours until equilibrated. Brains were embedded in optimal cutting temperature compound (OCT; Tissue-Tek, Sakura, Netherlands) and frozen in isopentane on dry ice. Coronal cryosections $(10 \mu \mathrm{m})$ were stored at $-20 \mathrm{C}$ prior to performing immunofluorescence. Protein expression for the expressed human LT $\alpha$ transgene was measured in snap-frozen rat CSF using a human TNF-beta/TNFSF1 Quantikine ELISA kit (DTBO0, R\&D systems) as per manufacturer's instructions.

\section{Immunohistochemistry and immunofluorescence}

Immunofluorescence was performed as previously described (James et al., 2020). For immunohistochemistry snap-frozen sections sections were fixed with cold-methanol $\left(4^{\circ} \mathrm{C}\right)$ for 10 minutes and blocked with 10\% normal horse serum (NHS), followed by overnight incubation with primary antibody, and then incubated with ImmPRESS HRP-conjugated secondary antibodies (Vector laboratories). For single IHC, slides were visualised with ImmPACT-DAB (Vector laboratories) as the chromagen. Dual colour IHC was performed by sequential staining, followed by detection with ImmPACT-DAB, incubation with the second primary antibody and detected using the $A B C$-alkaline phosphatase detection system with Vector blue as substrate. The primary antibodies used were: mouse anti-MOG (clone Y10, Prof Reynolds, Imperial College London, UK); rabbit anti-myelin basic protein (MBP) (Polyclonal, Merck, Darmstadt, Germany); mouse anti-neurofilament-H protein (clone NE14; Merck, Darmstadt, Germany); rabbit anti-IBA1 (Polyclonal IgG, Wako Pure Chemical Corporation, USA); rabbit anti-Glial Fibrillary Acidic Protein (GFAP) (Polyclonal, Dako Agilent, Santa Clara, CA, USA); mouse anti-NeuN (clone A60, Merck, Darmstadt, Germany); mouse anti-CD79a (ThermoFisher); mouse anti-CD4 (AbD Serotec); mouse anti-CD8 (AbD Serotec) goat anti-human LTA (R\&D systems); rabbit anti-laminin (Wako, Japan); rabbit anti-cleaved caspase-3 (Cell signalling, USA); goat anti-CXCL13 (R\&D systems); mouse anti-CCL21 (R\&D systems); mouse anti-CCL19 (R\&D systems); mouse anti-CXCL12 (monoclonal, Sigma UK); rabbit anti-CXCR4 (Sigma, UK); goat anti-LTBR (polyclonal Goat, R\&D); rabbit anti-Ki67 (Santa-Cruz); rabbit anti-CD3 (DAKO); rabbit anti-IgG (R\&D); rabbit anti-laminin (Novusbio); 
mouse anti-MadCam (monoclonal mouse, R\&D systems); mouser anti-CNPase (Millipore); rabbit anti-CXCR5 (Abcam, UK), rabbit anti-podoplanin (Abcam, UK); mouse anti-CCR7 (mouse monoclonal, R\&D systems).

All the secondary antibodies used for immunofluorescence were purchased from ThermoFisher Scientific (USA): Alexa Fluor 546 Goat Anti-Mouse IgG $(\mathrm{H}+\mathrm{L})$, Alexa Fluor 546 Goat Anti-Rabbit IgG(H+L), Alexa Fluor 488 Goat Anti-Mouse IgG $(\mathrm{H}+\mathrm{L})$ Fluor 488 Goat AntiRabbit IgG $(\mathrm{H}+\mathrm{L})$, Alexa Fluor 488 Goat Anti-Mouse IgG1, Alexa Fluor 546 Goat Anti-Mouse IgG1, Alexa Fluor 647 Goat Anti-Mouse IgG1, Alexa Fluor 488 Goat Anti-Mouse IgG2b, Alexa Fluor 647 Goat Anti-Mouse IgG2b, Alexa Fluor 488 Streptavidin, Alexa Fluor 546 Streptavidin, Alexa Fluor 647 Streptavidin. To couple with Alexa Fluor labelled Streptavidin, the following biotinylated antibodies were used from Vector Laboratories (UK): biotinylated goat anti-rabbit IgG $(\mathrm{H}+\mathrm{L})$, biotinylated horse anti-mouse $\lg G(\mathrm{H}+\mathrm{L})$ and biotinylated goat anti-mouse IgG2a (Life Technologies, ThermoFisher Scientific).

\section{Image acquisition}

Immunostained sections were viewed with either an epifluorescence Olympus BX63 scanning microscope or a SP8 Leica confocal microscope for the rat sections and a Zeiss Axioscope 5 for the human sections. Tiled digital images were obtained at $x 10$ or $\times 20$ magnification and coded for blinded analysis using ImageJ software (NIH, Maryland, USA). Higher magnification images were acquired on Leica Confocal at 60x magnification in $8 \mu \mathrm{m} z$-stacks.

For each animal, immunofluorescence tiled images were acquired from $4 \times 10 \mu \mathrm{m}$ sections stained with anti-MOG antibody to quantify demyelination. Cortical and midline layers were delineated as regions of interest (ROI) and automated Otsu thresholding was used to calculate the percentage area of ROI stained by the MOG antibody. For recombinant protein study thresholding was also used to calculate the area of IBA1 immunoreactivity (IR). For quantification of meningeal inflammation images of the length of the sagittal sulcus were acquired at 20x. DAPI staining was used to calculate the area of the subarachnoid space from the base of the sagittal sulcus to the position of the lateral blood vessels visible in the meninges. Meningeal immune cell numbers were counted manually for CD4, CD8 and CD79a expressing cells using the cell counting tool in ImageJ software and expressed as 
cells $/ \mathrm{mm}^{2}$, from $4 \times 10 \mu \mathrm{m}$ sequential sections spanning a $80 \mu \mathrm{m}$ distance in the anterior/posterior plane.

For both neuronal and microglial numbers, regions of interest were drawn that outlined the cortical layers or midline regions using ImageJ. Cortical layers were determined using costaining with DAPI and GluR2/3 to identify different cortical regions (Gardner et al., 2013; James et al., 2020). Cortical regions began from the lateral vessels in the meninges at the top of the sagittal sulcus and extended the breadth of the cortex to the rostral regions. $\mathrm{NeuN}+$ and IBA1+ cell numbers were counted manually at $20 \mathrm{x}$ magnification using the ImageJ cell counter tool. The total number of cells for all the counts was then divided by the total area of the region of interest to give the total cells $/ \mathrm{mm}^{2}$. For neurons and microglia, the number of cells was calculated from four sections per animal that were spaced $10 \mu \mathrm{m}$ apart covering a total distance of $80 \mu \mathrm{m}$ per animal.

\section{Statistical analysis}

Graphpad Prism8 statistical software (La Jolla, CA, USA) was used in all cases to present the data and to conduct statistical analysis. All data on graphs are expressed as mean \pm SEM except for PCR data which is shown as $\triangle \Delta C T$ with standard errors. Group comparisons for cell quantifications were analysed by one-way ANOVA with Tukey test for multiple comparisons.

\section{Online supplemental material}

Fig. S1 shows the testing and efficiency of the lentiviral vectors expressing human LT $\alpha$ eGFP following transduction by primary rat meningeal cells in culture. Table S1 lists the demographics and clinical characteristics of the post-mortem MS and control cases used for CSF and tissue analysis. Table S2 lists the demographics and clinical characteristics of the MS and control patient cohorts used for CSF analysis, as previously presented in Magliozzi et al., 2018.

\section{Acknowledgements.}


The authors would like to thank the Central Biomedical Services Staff at Imperial College for their help with animal welfare. This work was supported by the Multiple Sclerosis Society of Great Britain (grant 978/12 to RR and NDM and 037/15 to RR and REJ). NDM was supported by the European Research Council (7th Framework Proof of Concept grant no. 620253). EB and RS were supported by a PhD studentship from the UK Medical Research Council.

Author contributions: R. James contributed to the concept and design, acquisition, analysis and interpretations of the data and drafting of manuscript. E. Browne contributed to concept and design, acquisition, analysis and interpretations of the data and drafting of manuscript. R. Schalks contributed to concept and design, acquisition and analysis of data. L. Tan, P. Parakesh and H. Jacobs contributed to acquisition and analysis of data. R. Magliozzi contributed to analysis of human tissues and revision of the manuscript. M. Calabrese contributed to the clinical analysis of the patient cohort. N.D. Mazarakis contributed to concept and design, obtaining research grants and revision of the manuscript. R. Reynolds contributed to concept and design, acquisition, obtaining research grants, interpretation of data and drafting and revision of the manuscript.

Disclosures: The authors declare no competing financial interests.

\section{References}

Arpin, D.J., J.E. Gehringer, T.W. Wilson, and M.J. Kurz. 2017a. A reduced somatosensory gating response in individuals with multiple sclerosis is related to walking impairment. J Neurophysiol 118:2052-2058.

Arpin, D.J., E. Heinrichs-Graham, J.E. Gehringer, R. Zabad, T.W. Wilson, and M.J. Kurz. 2017b. Altered sensorimotor cortical oscillations in individuals with multiple sclerosis suggests a faulty internal model. Hum Brain Mapp 38:4009-4018.

Bar-Or, A., L. Fawaz, B. Fan, P.J. Darlington, A. Rieger, C. Ghorayeb, P.A. Calabresi, E. Waubant, S.L. Hauser, J. Zhang, and C.H. Smith. 2010. Abnormal B-cell cytokine responses a trigger of T-cell-mediated disease in MS? Ann Neurol 67:452-461. 
Bell, L., A. Lenhart, A. Rosenwald, C.M. Monoranu, and F. Berberich-Siebelt. 2019. Lymphoid Aggregates in the CNS of Progressive Multiple Sclerosis Patients Lack Regulatory $T$ Cells. Front Immunol 10:3090.

Benezech, C., A. White, E. Mader, K. Serre, S. Parnell, K. Pfeffer, C.F. Ware, G. Anderson, and J.H. Caamano. 2010. Ontogeny of stromal organizer cells during lymph node development. J Immunol 184:4521-4530.

Bevan, R.J., R. Evans, L. Griffiths, L.M. Watkins, M.I. Rees, R. Magliozzi, I. Allen, G. McDonnell, R. Kee, M. Naughton, D.C. Fitzgerald, R. Reynolds, J.W. Neal, and O.W. Howell. 2018. Meningeal inflammation and cortical demyelination in acute multiple sclerosis. Ann Neurol 84:829-842.

Browning, J.L., N. Allaire, A. Ngam-Ek, E. Notidis, J. Hunt, S. Perrin, and R.A. Fava. 2005. Lymphotoxin-beta receptor signaling is required for the homeostatic control of HEV differentiation and function. Immunity 23:539-550.

Buckle, G.J., P. Hollsberg, and D.A. Hafler. 2003. Activated CD8+ T cells in secondary progressive MS secrete lymphotoxin. Neurology 60:702-705.

Calabrese, M., A. Gajofatto, F. Gobbin, G. Turri, S. Richelli, A. Matinella, E.S. Oliboni, M.D. Benedetti, and S. Monaco. 2015a. Late-onset multiple sclerosis presenting with cognitive dysfunction and severe cortical/infratentorial atrophy. Mult Scler 21:580589.

Calabrese, M., R. Magliozzi, O. Ciccarelli, J.J. Geurts, R. Reynolds, and R. Martin. 2015b. Exploring the origins of grey matter damage in multiple sclerosis. Nat Rev Neurosci 16:147-158.

Cannella, B., I.D. Sizing, C.D. Benjamin, J.L. Browning, and C.S. Raine. 1997. Antibodies to lymphotoxin alpha (LT alpha) and LT beta recognize different glial cell types in the central nervous system. J Neuroimmunol 78:172-179.

Carassiti, D., D.R. Altmann, N. Petrova, B. Pakkenberg, F. Scaravilli, and K. Schmierer. 2018. Neuronal loss, demyelination and volume change in the multiple sclerosis neocortex. Neuropathol Appl Neurobiol 44:377-390.

Christensen, A.D., S. Skov, and C. Haase. 2013. Local and systemic effects of co-stimulatory blockade using cytotoxic T lymphocyte antigen-4-immunoglobulin in dinitrofluorobenzene- and oxazolone-induced contact hypersensitivity in mice. Clin Exp Immunol 171:220-230. 
Cohen, M., A. Giladi, C. Raposo, M. Zada, B. Li, J. Ruckh, A. Deczkowska, B. Mohar, R. Shechter, R.G. Lichtenstein, I. Amit, and M. Schwartz. 2021. Meningeal lymphoid structures are activated under acute and chronic spinal cord pathologies. Life Sci Alliance 4:e202000907.

Corcione, A., S. Casazza, E. Ferretti, D. Giunti, E. Zappia, A. Pistorio, C. Gambini, G.L. Mancardi, A. Uccelli, and V. Pistoia. 2004. Recapitulation of B cell differentiation in the central nervous system of patients with multiple sclerosis. Proc Natl Acad SciU S A 101:11064-11069.

Dang, A.K., Y. Tesfagiorgis, R.W. Jain, H.C. Craig, and S.M. Kerfoot. 2015. Meningeal Infiltration of the Spinal Cord by Non-Classically Activated B Cells is Associated with Chronic Disease Course in a Spontaneous B Cell-Dependent Model of CNS Autoimmune Disease. Front Immunol 6:470.

De Togni, P., J. Goellner, N.H. Ruddle, P.R. Streeter, A. Fick, S. Mariathasan, S.C. Smith, R. Carlson, L.P. Shornick, J. Strauss-Schoenberger, and et al. 1994. Abnormal development of peripheral lymphoid organs in mice deficient in lymphotoxin. Science 264:703-707.

Dendrou, C.A., L. Fugger, and M.A. Friese. 2015. Immunopathology of multiple sclerosis. Nat Rev Immunol 15:545-558.

Drayton, D.L., X. Ying, J. Lee, W. Lesslauer, and N.H. Ruddle. 2003. Ectopic LT alpha beta directs lymphoid organ neogenesis with concomitant expression of peripheral node addressin and a HEV-restricted sulfotransferase. J Exp Med 197:1153-1163.

Duddy, M., M. Niino, F. Adatia, S. Hebert, M. Freedman, H. Atkins, H.J. Kim, and A. Bar-Or. 2007. Distinct effector cytokine profiles of memory and naive human B cell subsets and implication in multiple sclerosis. J Immunol 178:6092-6099.

Durrenberger, P.F., F.S. Fernando, R. Magliozzi, S.N. Kashefi, T.P. Bonnert, I. Ferrer, D. Seilhean, B. Nait-Oumesmar, A. Schmitt, P.J. Gebicke-Haerter, P. Falkai, E. Grunblatt, M. Palkovits, P. Parchi, S. Capellari, T. Arzberger, H. Kretzschmar, F. Roncaroli, D.T. Dexter, and R. Reynolds. 2012. Selection of novel reference genes for use in the human central nervous system: a BrainNet Europe Study. Acta Neuropathol 124:893903.

Etemadi, N., J.K. Holien, D. Chau, G. Dewson, J.M. Murphy, W.S. Alexander, M.W. Parker, J. Silke, and U. Nachbur. 2013. Lymphotoxin alpha induces apoptosis, necroptosis and 
inflammatory signals with the same potency as tumour necrosis factor. FEBS $J$ 280:5283-5297.

Fernandes Filho, J.A., C.A. Vedeler, K.M. Myhr, H. Nyland, and J.P. Pandey. 2002. TNF-alpha and -beta gene polymorphisms in multiple sclerosis: a highly significant role for determinants in the first intron of the TNF-beta gene. Autoimmunity 35:377-380.

Fisniku, L.K., D.T. Chard, J.S. Jackson, V.M. Anderson, D.R. Altmann, K.A. Miszkiel, A.J. Thompson, and D.H. Miller. 2008. Gray matter atrophy is related to long-term disability in multiple sclerosis. Ann Neurol 64:247-254.

Gallego-Delgado, P., R. James, E. Browne, J. Meng, S. Umashankar, L. Tan, C. Picon, N.D. Mazarakis, A.A. Faisal, O.W. Howell, and R. Reynolds. 2020. Neuroinflammation in the normal-appearing white matter (NAWM) of the multiple sclerosis brain causes abnormalities at the nodes of Ranvier. PLOS Biol 18:e3001008.

Gardner, C., R. Magliozzi, P.F. Durrenberger, O.W. Howell, J. Rundle, and R. Reynolds. 2013. Cortical grey matter demyelination can be induced by elevated pro-inflammatory cytokines in the subarachnoid space of MOG-immunized rats. Brain 136:3596-3608.

GeurtsvanKessel, C.H., M.A. Willart, I.M. Bergen, L.S. van Rijt, F. Muskens, D. Elewaut, A.D. Osterhaus, R. Hendriks, G.F. Rimmelzwaan, and B.N. Lambrecht. 2009. Dendritic cells are crucial for maintenance of tertiary lymphoid structures in the lung of influenza virus-infected mice. J Exp Med 206:2339-2349.

Gommerman, J.L., and J.L. Browning. 2003. Lymphotoxin/light, lymphoid microenvironments and autoimmune disease. Nat Rev Immunol 3:642-655.

Gommerman, J.L., K. Giza, S. Perper, I. Sizing, A. Ngam-Ek, C. Nickerson-Nutter, and J.L. Browning. 2003. A role for surface lymphotoxin in experimental autoimmune encephalomyelitis independent of LIGHT. J Clin Invest 112:755-767.

Griffiths, D.R., T.M. Jenkins, C.P. Addington, S.E. Stabenfeldt, and J. Lifshitz. 2020. Extracellular matrix proteins are time-dependent and regional-specific markers in experimental diffuse brain injury. Brain Behav 10:e01767.

Haider, L., T. Zrzavy, S. Hametner, R. Hoftberger, F. Bagnato, G. Grabner, S. Trattnig, S. Pfeifenbring, W. Bruck, and H. Lassmann. 2016. The topograpy of demyelination and neurodegeneration in the multiple sclerosis brain. Brain 139:807-815.

Harrison, D.M., S. Roy, J. Oh, I. Izbudak, D. Pham, S. Courtney, B. Caffo, C.K. Jones, P. van Zijl, and P.A. Calabresi. 2015. Association of Cortical Lesion Burden on 7-T Magnetic 
Resonance Imaging With Cognition and Disability in Multiple Sclerosis. JAMA Neurol 72:1004-1012.

Herzog, B.H., J. Fu, S.J. Wilson, P.R. Hess, A. Sen, J.M. McDaniel, Y. Pan, M. Sheng, T. Yago, R. Silasi-Mansat, S. McGee, F. May, B. Nieswandt, A.J. Morris, F. Lupu, S.R. Coughlin, R.P. McEver, H. Chen, M.L. Kahn, and L. Xia. 2013. Podoplanin maintains high endothelial venule integrity by interacting with platelet CLEC-2. Nature 502:105-109.

Hirose, T., Y. Fukuma, A. Takeshita, and K. Nishida. 2018. The role of lymphotoxin-alpha in rheumatoid arthritis. Inflamm Res 67:495-501.

Howell, O.W., C.A. Reeves, R. Nicholas, D. Carassiti, B. Radotra, S.M. Gentleman, B. Serafini, F. Aloisi, F. Roncaroli, R. Magliozzi, and R. Reynolds. 2011. Meningeal inflammation is widespread and linked to cortical pathology in multiple sclerosis. Brain 134:27552771.

Howell, O.W., E.K. Schulz-Trieglaff, D. Carassiti, S.M. Gentleman, R. Nicholas, F. Roncaroli, and R. Reynolds. 2015. Extensive grey matter pathology in the cerebellum in multiple sclerosis is linked to inflammation in the subarachnoid space. Neuropathol Appl Neurobiol 41:798-813.

James, R.E., R. Schalks, E. Browne, I. Eleftheriadou, C.P. Munoz, N.D. Mazarakis, and R. Reynolds. 2020. Persistent elevation of intrathecal pro-inflammatory cytokines leads to multiple sclerosis-like cortical demyelination and neurodegeneration. Acta Neuropathol Commun 8:66.

Kain, M.J., and B.M. Owens. 2013. Stromal cell regulation of homeostatic and inflammatory lymphoid organogenesis. Immunology 140:12-21.

Kerschensteiner, M., C. Stadelmann, B.S. Buddeberg, D. Merkler, F.M. Bareyre, D.C. Anthony, C. Linington, W. Bruck, and M.E. Schwab. 2004. Targeting experimental autoimmune encephalomyelitis lesions to a predetermined axonal tract system allows for refined behavioral testing in an animal model of multiple sclerosis. American Journal of Pathology 164:1455-1469.

Kratz, A., A. Campos-Neto, M.S. Hanson, and N.H. Ruddle. 1996. Chronic inflammation caused by lymphotoxin is lymphoid neogenesis. J Exp Med 183:1461-1472.

Krautler, N.J., V. Kana, J. Kranich, Y. Tian, D. Perera, D. Lemm, P. Schwarz, A. Armulik, J.L. Browning, M. Tallquist, T. Buch, J.B. Oliveira-Martins, C. Zhu, M. Hermann, U. 
Wagner, R. Brink, M. Heikenwalder, and A. Aguzzi. 2012. Follicular dendritic cells emerge from ubiquitous perivascular precursors. Cell 150:194-206.

Kuerten, S., A. Schickel, C. Kerkloh, M.S. Recks, K. Addicks, N.H. Ruddle, and P.V. Lehmann. 2012. Tertiary lymphoid organ development coincides with determinant spreading of the myelin-specific T cell response. Acta Neuropathol 124:861-873.

Kutzelnigg, A., C.F. Lucchinetti, C. Stadelmann, W. Bruck, H. Rauschka, M. Bergmann, M. Schmidbauer, J.E. Parisi, and H. Lassmann. 2005. Cortical demyelination and diffuse white matter injury in multiple sclerosis. Brain 128:2705-2712.

Lindhout, E., M. van Eijk, M. van Pel, J. Lindeman, H.J. Dinant, and C. de Groot. 1999. Fibroblast-like synoviocytes from rheumatoid arthritis patients have intrinsic properties of follicular dendritic cells. J Immunol 162:5949-5956.

Lucchinetti, C.F., B.F. Popescu, R.F. Bunyan, N.M. Moll, S.F. Roemer, H. Lassmann, W. Bruck, J.E. Parisi, B.W. Scheithauer, C. Giannini, S.D. Weigand, J. Mandrekar, and R.M. Ransohoff. 2011. Inflammatory cortical demyelination in early multiple sclerosis. $N$ Engl J Med 365:2188-2197.

Madsen, P.M., B.H. Clausen, M. Degn, S. Thyssen, L.K. Kristensen, M. Svensson, N. Ditzel, B. Finsen, T. Deierborg, R. Brambilla, and K.L. Lambertsen. 2016a. Genetic ablation of soluble tumor necrosis factor with preservation of membrane tumor necrosis factor is associated with neuroprotection after focal cerebral ischemia. J Cereb Blood Flow Metab 36:1553-1569.

Madsen, P.M., D. Motti, S. Karmally, D.E. Szymkowski, K.L. Lambertsen, J.R. Bethea, and R. Brambilla. 2016b. Oligodendroglial TNFR2 Mediates Membrane TNF-Dependent Repair in Experimental Autoimmune Encephalomyelitis by Promoting Oligodendrocyte Differentiation and Remyelination. J Neurosci 36:5128-5143.

Magliozzi, R., S. Columba-Cabezas, B. Serafini, and F. Aloisi. 2004. Intracerebral expression of CXCL13 and BAFF is accompanied by formation of lymphoid follicle-like structures in the meninges of mice with relapsing experimental autoimmune encephalomyelitis. $J$ Neuroimmunol 148:11-23.

Magliozzi, R., O. Howell, A. Vora, B. Serafini, R. Nicholas, M. Puopolo, R. Reynolds, and F. Aloisi. 2007. Meningeal B-cell follicles in secondary progressive multiple sclerosis associate with early onset of disease and severe cortical pathology. Brain 130:10891104. 
Magliozzi, R., O.W. Howell, R. Nicholas, C. Cruciani, M. Castellaro, C. Romualdi, S. Rossi, M. Pitteri, M.D. Benedetti, A. Gajofatto, F.B. Pizzini, S. Montemezzi, S. Rasia, R. Capra, A. Bertoldo, F. Facchiano, S. Monaco, R. Reynolds, and M. Calabrese. 2018. Inflammatory intrathecal profiles and cortical damage in multiple sclerosis. Ann Neurol 83:739-755.

Magliozzi, R., O.W. Howell, C. Reeves, F. Roncaroli, R. Nicholas, B. Serafini, F. Aloisi, and R. Reynolds. 2010. A Gradient of neuronal loss and meningeal inflammation in multiple sclerosis. Ann Neurol 68:477-493.

Magliozzi, O.W. Howell, P. Durrenberger, E. Arico, R. James, C. Cruciani, C. Reeves, F. Roncaroli, R. Nicholas, and R. Reynolds. 2019. Meningeal inflammation changes the balance of TNF signalling in cortical grey matter in multiple sclerosis. J Neuroinflammation, 16:259

Magliozzi, R., A. Scalfari, A.I. Pisani, S. Ziccardi, D. Marastoni, F.B. Pizzini, A. Bajrami, A. Tamanti, M. Guandalini, S. Bonomi, S. Rossi, V. Mazziotti, M. Castellaro, S. Montemezzi, S. Rasia, R. Capra, M. Pitteri, C. Romualdi, R. Reynolds, and M. Calabrese. 2020. The CSF Profile Linked to Cortical Damage Predicts Multiple Sclerosis Activity. Ann Neurol 88:562-573.

Matusevicius, D., V. Navikas, M. Soderstrom, B.G. Xiao, M. Haglund, S. Fredrikson, and H. Link. 1996. Multiple sclerosis: the proinflammatory cytokines lymphotoxin-alpha and tumour necrosis factor-alpha are upregulated in cerebrospinal fluid mononuclear cells. J Neuroimmunol 66:115-123.

McWilliam, O., F. Sellebjerg, H.V. Marquart, and M.R. von Essen. 2018. B cells from patients with multiple sclerosis have a pathogenic phenotype and increased LTalpha and TGFbeta1 response. J Neuroimmunol 324:157-164.

Medvedev, A.E., T. Espevik, G. Ranges, and A. Sundan. 1996. Distinct roles of the two tumor necrosis factor (TNF) receptors in modulating TNF and lymphotoxin alpha effects. $J$ Biol Chem 271(Suppl 16):9778-9784.

Merkler, D., T. Ernsting, M. Kerschensteiner, W. Bruck, and C. Stadelmann. 2006. A new focal EAE model of cortical demyelination: multiple sclerosis-like lesions with rapid resolution of inflammation and extensive remyelination. Brain 129:1972-1983.

Mitsdoerffer, M., and A. Peters. 2016. Tertiary Lymphoid Organs in Central Nervous System Autoimmunity. Front Immunol 7:451. 
Navikas, V., B. He, J. Link, M. Haglund, M. Soderstrom, S. Fredrikson, A. Ljungdahl, J. Hojeberg, J. Qiao, T. Olsson, and H. Link. 1996. Augmented expression of tumour necrosis factor-alpha and lymphotoxin in mononuclear cells in multiple sclerosis and optic neuritis. Brain 119:213-223.

Ofengeim, D., and J. Yuan. 2013. Regulation of RIP1 kinase signalling at the crossroads of inflammation and cell death. Nat Rev Mol Cell Biol 14:727-736.

Papadopoulos, D., D. Pham-Dinh, and R. Reynolds. 2006. Axon loss is responsible for chronic neurological deficit following inflammatory demyelination in the rat. Exp Neurol 197:373-385.

Perros, F., P. Dorfmuller, D. Montani, H. Hammad, W. Waelput, B. Girerd, N. Raymond, O. Mercier, S. Mussot, S. Cohen-Kaminsky, M. Humbert, and B.N. Lambrecht. 2012. Pulmonary lymphoid neogenesis in idiopathic pulmonary arterial hypertension. Am J Respir Crit Care Med 185:311-321.

Peters, A., L.A. Pitcher, J.M. Sullivan, M. Mitsdoerffer, S.E. Acton, B. Franz, K. Wucherpfennig, S. Turley, M.C. Carroll, R.A. Sobel, E. Bettelli, and V.K. Kuchroo. 2011. Th17 cells induce ectopic lymphoid follicles in central nervous system tissue inflammation. Immunity 35:986-996.

Picon, C., A. Jayaraman, R. James, C. Beck, P. Gallego, M.E. Witte, J. van Horssen, N.D. Mazarakis, and R. Reynolds. 2021. Neuron-specific activation of necroptosis signaling in multiple sclerosis cortical grey matter. Acta Neuropathol 141:585-604.

Pikor, N.B., J.L. Astarita, L. Summers-Deluca, G. Galicia, J. Qu, L.A. Ward, S. Armstrong, C.X. Dominguez, D. Malhotra, B. Heiden, R. Kay, V. Castanov, H. Touil, L. Boon, P. O'Connor, A. Bar-Or, A. Prat, V. Ramaglia, S. Ludwin, S.J. Turley, and J.L. Gommerman. 2015. Integration of Th17- and Lymphotoxin-Derived Signals Initiates Meningeal-Resident Stromal Cell Remodeling to Propagate Neuroinflammation. Immunity 43:1160-1173.

Probert, L. 2015. TNF and its receptors in the CNS: The essential, the desirable and the deleterious effects. Neuroscience 302:2-22.

Raine, C.S., B. Bonetti, and B. Cannella. 1998. Multiple sclerosis: expression of molecules of the tumor necrosis factor ligand and receptor families in relationship to the demyelinated plaque. Rev Neurol (Paris) 154:577-585. 
Raphael, I., F. Gomez-Rivera, R.A. Raphael, R.R. Robinson, S. Nalawade, and T.G. Forsthuber. 2019. TNFR2 limits proinflammatory astrocyte functions during EAE induced by pathogenic DR2b-restricted T cells. J Clin Invest Insight 4:

Reali, C., R. Magliozzi, F. Roncaroli, R. Nicholas, O.W. Howell, and R. Reynolds. 2020. B cell rich meningeal inflammation associates with increased spinal cord pathology in multiple sclerosis. Brain Pathol 30:779-793.

Reynolds, R., F. Roncaroli, R. Nicholas, B. Radotra, D. Gveric, and O. Howell. 2011. The neuropathological basis of clinical progression in multiple sclerosis. Acta Neuropathol 122:155-170.

Rieckmann, P., M. Albrecht, B. Kitze, T. Weber, H. Tumani, A. Broocks, W. Luer, A. Helwig, and S. Poser. 1995. Tumor necrosis factor-alpha messenger RNA expression in patients with relapsing-remitting multiple sclerosis is associated with disease activity. Ann Neurol 37:82-88.

Rodriguez, E.G., C. Wegner, M. Kreutzfeldt, K. Neid, D.R. Thal, T. Jurgens, W. Bruck, C. Stadelmann, and D. Merkler. 2014. Oligodendroglia in cortical multiple sclerosis lesions decrease with disease progression, but regenerate after repeated experimental demyelination. Acta Neuropathol 128:231-246.

Romme Christensen, J., L. Bornsen, R. Ratzer, F. Piehl, M. Khademi, T. Olsson, P.S. Sorensen, and F. Sellebjerg. 2013. Systemic inflammation in progressive multiple sclerosis involves follicular T-helper, Th17- and activated B-cells and correlates with progression. PLoS One 8:e57820.

Selmaj, K., C.S. Raine, B. Cannella, and C.F. Brosnan. 1991a. Identification of lymphotoxin and tumor necrosis factor in multiple sclerosis lesions. J Clin Invest 87:949-954.

Selmaj, K., C.S. Raine, M. Farooq, W.T. Norton, and C.F. Brosnan. 1991b. Cytokine cytotoxicity against oligodendrocytes. Apoptosis induced by lymphotoxin. J Immunol 147:1522-1529.

Serafini, B., B. Rosicarelli, R. Magliozzi, E. Stigliano, and F. Aloisi. 2004. Detection of ectopic B-cell follicles with germinal centers in the meninges of patients with secondary progressive multiple sclerosis. Brain Pathol 14:164-174.

Stadelmann, C. 2011. Multiple sclerosis as a neurodegenerative disease: pathology, mechanisms and therapeutic implications. Curr Opin Neurol 24:224-229. 
Strik, M., D.T. Chard, I. Dekker, K.A. Meijer, A.J. Eijlers, M. Pardini, B.M. Uitdehaag, S.C. Kolbe, J.J. Geurts, and M.M. Schoonheim. 2020. Increased functional sensorimotor network efficiency relates to disability in multiple sclerosis. Mult Scler DOI: $10.1177 / 1352458520966292$.

Suen, W.E., C.M. Bergman, P. Hjelmstrom, and N.H. Ruddle. 1997. A critical role for lymphotoxin in experimental allergic encephalomyelitis. J Exp Med 186:1233-1240.

Taoufik, E., V. Tseveleki, S.Y. Chu, T. Tselios, M. Karin, H. Lassmann, D.E. Szymkowski, and L. Probert. 2011. Transmembrane tumour necrosis factor is neuroprotective and regulates experimental autoimmune encephalomyelitis via neuronal nuclear factorkappaB. Brain 134:2722-2735.

Trapp, B.D., M. Vignos, J. Dudman, A. Chang, E. Fisher, S.M. Staugaitis, H. Battapady, S. Mork, D. Ontaneda, S.E. Jones, R.J. Fox, J. Chen, K. Nakamura, and R.A. Rudick. 2018. Cortical neuronal densities and cerebral white matter demyelination in multiple sclerosis: a retrospective study. Lancet Neurol 17:870-884.

van Nierop, K., and C. de Groot. 2002. Human follicular dendritic cells: function, origin and development. Semin Immunol 14:251-257.

Watkins, L.M., J.W. Neal, S. Loveless, I. Michailidou, V. Ramaglia, M.I. Rees, R. Reynolds, N.P. Robertson, B.P. Morgan, and O.W. Howell. 2016. Complement is activated in progressive multiple sclerosis cortical grey matter lesions. J Neuroinflammation $13: 161$.

Yang, K., Y. Liang, Z. Sun, L. Liu, J. Liao, H. Xu, M. Zhu, Y.X. Fu, and H. Peng. 2018. T cellderived lymphotoxin limits Th1 response during HSV-1 infection. Sci Rep 8:17727.

Yates, R.L., M.M. Esiri, J. Palace, B. Jacobs, R. Perera, and G.C. DeLuca. 2017. Fibrin(ogen) and neurodegeneration in the progressive multiple sclerosis cortex. Ann Neurol 82:259-270. 


\section{Figure legends}

Figure 1. Analysis of the levels of LT $\alpha$ gene transcript and protein. (A) Levels of LT $\alpha$ measured in diagnostic CSF samples from 10 non-inflammatory neurological controls, 18 MSHigh and 13 MSLow cases by Bioplex analysis. The number of cortical lesions on 3D DIR MRI was used to stratify naive MS patients as MSHigh (greater than 10 cortical lesions) or MSlow inflammation (less than 2 cortical lesions). (B) LT $\alpha$ protein levels in post-mortem CSF from 10 MSHigh, 10 MSLow and 10 control cases, measured by electrochemoluminescence (MSD). SPMS post-mortem cases were classed as MSHigh or MSLow based on the degree of immune cell infiltration in the meninges, based on the numbers of haematoxylin nuclei in the infiltrate. (C) LTa gene transcript levels in meninges dissected from post-mortem tissue blocks of 16 SPMS cases and 8 controls, determined by QPCR. Graph shows fold change in mRNA levels in MS versus control. (D) Immunostaining for LT $\alpha$ in meningeal aggregates at 10x shows cytoplasmic staining in numerous cells in meningeal infiltrates. Scale bar $=50 \mu \mathrm{m}$. (E) Co-staining for lymphotoxin- $\alpha$ (blue) and CD3 (brown) in meninges show double positive staining on CD3+ T-cells. In addition, some LT $\alpha$ expressing cells are not CD3+ T-cells (yellow stars). Scale bar $=50 \mu \mathrm{m}$. (F-G) LT $\alpha$ (blue) and CD3 (brown) co-staining at higher magnification. Scale bar=20 $\mu \mathrm{m}$. (H) Immunostaining for LTRR in meningeal aggregates shows a reticular like staining. Statistics: 1-way analysis of variance with Tukey post-test.

Figure 2. Injection of recombinant LT $\alpha$ and IFN $\gamma$ into the subarachnoid space of rats leads to meningeal inflammation. (A) Cytokine injection into the SS led to the formation of large dense infiltrates of cells the length of the sagittal sulcus and across the surface of the cortex visualised here at $4 x$ magnification by DAPI immunostaining. Injection of PBS did not lead to any infiltration. Scale bar $=100 u m$. (B) Representative images of CD4, CD8 and CD79a from sequential sections at $3 \mathrm{dpi}$ post injection of cytokines. Scale bar 50= $\mu \mathrm{m}$. (C-D) Quantification of CD4+ (C) and CD8+ (D) cell numbers in the SS expressed as cells $/ \mathrm{mm}^{2}$. Both CD4+ and CD8+ numbers were maximal at 3dpi with CD4+ cell number 104\% higher than CD8+. 14 and $21 \mathrm{dpi}$ were not significantly increased compared to naïve. Data presented as mean \pm SEM ( $n=3-4$ per group). ${ }^{* * * *} \mathrm{P}<0.0001$ cytokine compared to naïve. (E) Quantification of CD79a cells expressed as cells $/ \mathrm{mm}^{2}$ in the sagittal sulcus. The maximal infiltration of CD79a+ B-cells into the sagittal sulcus was seen at 7dpi and was significantly highly than naive at 3,14 and 
21dpi. * $\mathrm{p}<0.05 ; * * \mathrm{p}<0.001, * * * * \mathrm{p}<0.0001$ cytokine vs naïve. (F) CD79a+ B-cells formed densely packed, focal clusters of cells within the meningeal aggregates. Scale bar=100 $\mu \mathrm{m}$. (G) Following cytokine injection a proportion of CD79a+ cells (green) were positive for the proliferation marker Ki67 (red). (H) Data showing the percentage of CD79a+ cells that costained with Ki67. At 3 dpi $65 \%$ of cells were double positive $(n=4)$, significantly decreasing to $37.7 \%$ at $7 \mathrm{dpi}(n=4), 7.5 \%$ at $14 \mathrm{dpi}(n=3)$ and $0.07 \%$ at $21 \mathrm{dpi}(n=3)$. Statistics: 1 -way analysis of variance with Tukey post-test. ${ }^{*} p<0.05, * * * p<0.001, * * * * p<0.0001$ compared to $3 d p i$.

Figure 3. Subpial demyelination following injection of recombinant LT $\alpha+I F N \gamma$ into the subarachnoid space. (A-O) Immunofluorescence for myelin oligodendrocyte glycoprotein (MOG) and the microglial marker IBA1 in rmMOG immunised rats injected with PBS (A-C) or $1 \mathrm{mg} \mathrm{LT} \alpha$ and 75ng IFN $\gamma$ recombinant cytokines culled at 3 (D-F), 7 (G-I), 14 (J-L) and 21 (MO) days post-cytokine injection (dpi), demonstrating the degree of demyelination and microglial activation at various times points. Cortex and midline images are magnifications of the regions shown by the boxed areas in image A. Scale bars: $A=500 \mu \mathrm{m}, \mathrm{B}$ and $\mathrm{C}=50 \mu \mathrm{m}$. (P-Q) Quantification of subpial demyelination at the injection site in layer I (P) and layers IIIII (Q), presented as the percentage area covered by MOG immunofluorescence (IF) ( $n=3-4$ each group). Data is presented as the mean \pm SEM. Statistics: 1-way analysis of variance with Tukey post-test. * $\mathrm{P}<0.01 ; * * * \mathrm{p}<0.005, * * * * \mathrm{p}<0.001$ cytokine vs PBS; \# $\mathrm{p}<0.057$ vs $21 \mathrm{dpi}$. (R-S) Measurement of the area covered by IBA1 immunofluorescence in cortical layers I-III (R) and midline layers I-III (S) and presented at mean \pm SEM. * $p<0.05 ; * * p<0.001$ cytokine vs naïve.

Figure 4. Primary meningeal cells transduced with lentiviral vector expressing human LT $\alpha$. (A) Lentiviral vectors expressing eGFP as a control or LT $\alpha$ were injected into the sagittal sulcus. Following LV-LT $\alpha$ injection the human LT $\alpha$ transgene could be measured in the meninges dissected from rats at $90 \mathrm{dpi}$, demonstrating long term expression of the gene by the CMV promoter within the transduced meningeal cells ( $n=3$ rats), but no expression in the eGFP controls. Data shown as ratio change of $2^{\wedge} \Delta \Delta C T$ for hLT $\alpha$ mRNA in LVGFP and LVLT $\alpha$ compared to naive animals. (B) Significant transduction of meningeal cells with the lentiviral vectors could be demonstrated by expression of the GFP protein (green), the length of the SS and across the lateral surface of the cortex. Higher magnification image in box. (C) Immunostaining for $\mathrm{hLT} \alpha$ (red) showed expression of the human LT $\alpha$ protein along 
the length of the SS that was present both at 28 and 90 dpi demonstrating widespread transduction. (D) CSF samples were taken from the cisterna magna of injected animals shortly before perfusion at 28 and $90 \mathrm{dpi}$. hLT $\alpha$ levels were measured in $100 \mu \mathrm{I}$ CSF samples using electrochemiluminescence (MSD plates). hLT $\alpha$ could be detected at both $28 \mathrm{dpi}$ (mean $389.6 \mathrm{pg} / \mathrm{mL})$ and $90 \mathrm{dpi}(333.6 \mathrm{pg} / \mathrm{mL})$ at similar levels but was not present in LVGFP animals. Statistics: 1-way analysis of variance with Tukey post-test. **** $p<0.001$. (D) Quantification of $\mathrm{hLT} \alpha$ protein levels by ELISA in tissue samples dissected from either naïve or LVLTa injected animals at $90 \mathrm{dpi}$ at the site of injection and from the surrounding cerebral cortex and the cerebellum, demonstrating very local transduction.

\section{Figure 5. Overexpression of LT $\alpha$ in the subarachnoid space induces meningeal} inflammation. (A) DAPI nuclear staining showing the accumulation of cells down the entire length of the sagittal sulcus in both IFA and MOG immunised animals at 28 and $90 \mathrm{dpi}$. No accumulation of cells can be seen in GFP vector animals. Scale bar $=200 \mu \mathrm{m}$ (B) Immunostaining shows presence of CD4+ and CD8+ T-cells, CD79a+ B-cells and IBA1+ myeloid cells within the dense infiltrates. Scale bar $=100 \mu \mathrm{m}$. (C-D) Quantification of the numbers of CD4+ (C) and CD8+ (D) cells/mm2 down the length of the SAS in all animal groups. Data presented as mean \pm SEM ( $n=3-4$ per group). $* * * * P<0.001$ cytokine compared to naïve. (E) Quantification of CD79a+ B-cells expressed as cells $/ \mathrm{mm}^{2}$ in the sagittal sulcus shows no difference in numbers between IFA and MOG immunised animals and between 28 and 90 dpi (* p<0.05). (F) Immunostaining of a cluster of CD79a cells within the SS. (G) Ig expressing plasma cells were seen within CD79a+ B-cell clusters. $(\mathbf{H})$ Regions where there were dense clusters of CD79a+ cells showed very little presence of CD8 and CD4 cells, shown in a serial section. (I) The proportion of CD79a+ cells that were co-expressing the proliferation marker Ki67 (J) was similar for MOG and IFA immunised animals at both 28 and $90 \mathrm{dpi}$. (K) Dense aggregates of cells were also present across the surface of the cortex (scale bar=150 $\mu \mathrm{m}$ ), containing CD8+ and CD4+ T-cells (L) and clusters of CD79a+ B-cells (M), particularly around large channels or vessels. (N) CD4+ and CD8+ T-cells showed discrete localisation patterns that were distinct from CD79a+ cells. Statistics: 1-way analysis of variance with Tukey post-test. Scale bar $H, M, N=100 \mu \mathrm{m}$. Scale bar $\mathrm{G}, \mathrm{J}, \mathrm{L}=40 \mu \mathrm{m}$.

Figure 6. Presence of lymphatic channels in meningeal aggregates. (A) DAPI staining of meningeal aggregate at $28 \mathrm{dpi}$ in a MOG immunised animal showing the presence of 
multiple channels within the infiltrates that varied in size from small (red arrows) to very large, over $100 \mu \mathrm{m}$ in size (green arrows). (B) Immunostaining against MadCam-1 showed high expression the length of the SAS, particularly around the channels/vessels. Scale bar = 100um. (C-D) Higher magnification images show strong MadCaM immunofluorescence around large vessels/venules (C) as well as on a process bearing cellular network (D). (E) Laminin staining on the basement membranes surrounding SAS, illustrating the glia limitans boundary as well as around lymphatic vessels in both MOG and IFA immunised animals at 28 dpi. Laminin immunostaining on blood vessels was much weaker. (F) Podoplanin immunohistochemistry shows a reticulum of staining throughout the immune cell infiltrates. Scale bar $=50 \mu \mathrm{m}$. (G) Higher magnification images of podoplanin show IFA and MOG animals express similar staining patterns in the cytoplasm and processes of meningeal cells. Scale bar $=20 \mu \mathrm{m}$.

Figure 7. Expression lymphoid chemokines by meningeal cells. (A) Primary meningeal cells were treated with 100ng LT $\alpha$ for 24 hours and changes in gene expression measured by RTPCR. Ratio changes calculated for LT $\alpha$ treated over non-treated showed increased gene expression levels for CCL19, CXCL13 and LTBR. * $p<0.05$. (B) The cortical meninges were microdissected from $4 \times 10 \mu \mathrm{m}$ section from 28 dpi GFP or LVLT $\alpha$ injected animals for RNA extraction. Changes in mRNA levels for chemokines and receptors was measured by RT-PCR and shown here as Cytokine treated compared to GFP animals. ${ }^{*} \mathrm{p}<0.05 \mathrm{t}$-test on $\Delta \Delta \mathrm{Ct}$ values. (C) In the dissected meninges, CXCL13 transcript showed the greatest fold change in cytokine versus GFP animals at 28 dpi. ** p<0.01. (D) Immunostaining for CXCL13 at 28 dpi showed expression on blood vessels and lymphatic channels and on a network of cell processes through the immune cell infiltrate. Scale bar $=100 \mu \mathrm{m}$. (E) Higher magnification shows CXCL13+ staining on cells with follicular dendritic cell morphology with multiple thin processes. Scale bar $=40 \mu \mathrm{m}$. (F) IHC staining against ED5, a marker for follicular dendritic cells showed a dense network of cells through the infiltrates at $28 \mathrm{dpi}$. Inset box shows a higher magnification view. (G) CXCL13/CXCR5 double immunofluorescence and CD79a immunostaining on a serial section of cortical surface infiltrates. (H) Triple immunostaining for CCL21 and CXCL13 in regions with CD3+ T-cells showed distinct localisation patterns. (I) CXCL12 immunostaining was seen throughout the aggregates, together with expression of its receptor CXCR4 receptor (J). CCL21 and CCL19 double immunofluorescence in midline (K) 
and cortical aggregates (L). (M) Cells expressing CCR7 were found throughout the midline and cortical aggregates.

Figure 8. Demyelination after chronic overexpression of LT $\alpha$. (A-B) Representative images of MOG staining in LVLTa injected animals immunised with IFA or MOG at 28dpi (A) and 90dpi (B). Demyelination was present in subpial areas in rmMOG immunised rats at 28 and 90 days after LVLTa injection (delineated by dotted lines). (C-D) Quantification of subpial demyelination in midline layers I-III (C) and cortical layers I-III (D) presented as the percentage area covered by MOG immunofluorescence (IF). Data is presented as the mean \pm SEM. Statistics: 1-way analysis of variance with Tukey post-test. *** $\mathrm{P}<0.001 ; * * * * p<0.001$ cytokine vs naïve. Naïve and GFP groups $n=6$, LVLTa groups $n=8$. (E-F) Immunofluorescence for MOG at higher magnification shows no demyelination in eGFP vector injected animals compared to MOG immunised animals in layers I and II in the cortex (E) or midline (F) at 90 dpi. (G) MBP immunostaining confirmed the lack of myelin shown by MOG immunostaining. (H) IBA1 immunofluorescence revealed little microglial activation immediately surrounding the sagittal sulcus and subarachnoid space in MOG immunised animals at 90 days post LVGFP injection, whereas prominent microglial activation occurred throughout the corpus callosum and cortex following LVLTa injection. (I) Ameboid IBA1+ cells were found in layer VI compared to ramified IBA1+ cells in the surface cortex. (J) Quantification of IBA1 cells $/ \mathrm{mm}^{2}$ showed significant increases in IBA1 cells $/ \mathrm{mm}^{2}$ at 28 and $90 \mathrm{dpi}$ in midline layers. (K) In the surface cortical regions only MOG immunised animals showed a significant increase in IBA1+ cell numbers. There was no significant difference between IFA or MOG immunised animals. ${ }^{*} p<0.05, * * p<0.01, * * * p<0.001$. Statistics 1 -way ANOVA with Tukey post test.

Figure 9. Neuronal loss in the cortical parenchyma after 90 days persistent LT $\alpha$ expression.

(A) Representative images of NeuN immunofluorescence in midline layers I-III for naïve, LVGFP in IFA and MOG animals and LVLT $\alpha$ in IFA and MOG animals, showing a decreased neuronal density in LVLT $\alpha$ injected animals. Scale bar $=50 \mu \mathrm{m}$. (B) Quantification of NeuN+ cells per $\mathrm{mm}^{2}$ in midline layer I-III regions after LV injection. GFP animals had no decrease in NeuN+ cells whilst LV-LT $\alpha$ animals had significant decreases in neuronal numbers both in IFA (613.8 cells $\left./ \mathrm{mm}^{2}, \mathrm{n}=8\right)$ and MOG (609.6 cells $\left./ \mathrm{mm}^{2}, \mathrm{n}=8\right)$ immunised animals compared to naïve (924.83cells $/ \mathrm{mm}^{2}, \mathrm{n}=6$ ). Graph presented is mean $+/-$ SEM. $* * * * p<0.0001$. (C) 
Representative images of NeuN immunostaining in cortical layers I-III from motor cortex 90 days after cytokine or GFP vector injection. A decreased density of NeuN expressing neurons is seen in both IFA and MOG LV-LT $\alpha$ but not in GFP animals. Scale bar $=60 \mu \mathrm{m}$. (D) Graph showing mean number of $\mathrm{NeuN}+$ cells $/ \mathrm{mm}^{2}$ in cortical layers I-III in all animal groups. A significant loss of NeuN+ cell is shown in both LVLTa injected IFA and MOG immunised animals when compared to GFP animals. There was no significant difference between IFA and MOG immunised LVLT $\alpha$ injected animals. ${ }^{*} p<<0.01, * * * p<0.001, * * * * P<0.0001$ cytokine versus naive. $\Delta \Delta \mathrm{p}<0.0, \Delta \Delta \Delta \Delta \mathrm{p}<0.001$ IFA /MOG versus IFA/MOG. (E) Representative images of NeuN immunostaining in rostral cortical regions. Loss of NeuN immunostaining in LVLT $\alpha$ animals compared to naïve controls was most visibly apparent in rostral regions, with loss present in both IFA and MOG animals. One MOG animal had focal neuronal loss that extended from the pial surface down through to layer IV (star in final image). Scale bar $=100 \mu \mathrm{m}$. (F) Quantification of $\mathrm{NeuN}+$ cells $/ \mathrm{mm}^{2}$ in cortical layer V. $* * p<0.01, * * * * p<0.0001$ cytokine vs naïve. (G) NeuN+ staining in layer $V$ of naïve and LVLT $\alpha$ MOG animals. In layer $V$ there was a decrease in NeuN staining density, in particular for smaller size interneurons, rather than the larger pyramidal cells. (H) 200 kDa neurofilament protein immunofluorescence within the cortex in proximity to the midline showed a decreased density of staining in both IFA and MOG LVLT $\alpha$ animals compared to naive controls. Scale bar $=50 \mu \mathrm{m}$. Statistics -1 -way analysis of variance with Tukey post-test.

\section{Supplementary figure 1. Primary meningeal cells transduced with lentiviral vectors}

expressing human LT $\alpha$. Primary meningeal cells were produced from meninges dissected from P2 rat pup cortices and cultured for 3 weeks. (A) Around 20\% of cells expressed TNFR1 and (B) approximately $98 \%$ of cells expressed laminin. (C) Cells transduced with lentiviral vectors (LVs) expressing enhanced GFP (LVGFP) did not any obvious changes in cell morphology or signs of stress and show high levels of GFP expression at 72hours following transduction (D). (E) Immunostaining untreated control meningeal cells with an antibody specific to the human form of lymphotoxin alpha showed no constitutive expression. (F) Following transduction with LV-LT $\alpha$ at an $\mathrm{MOI}$ of 1000 , immunostaining with a hLT $\alpha$ antibody showed a transduction efficiency of around $98 \% 72$ hours post-transduction. Cleaved caspase-3 staining showed apoptotic cells were rare (G). (H) LTo expression by human embryonic kidney 293T (HEK) cells at 24 (H) or 48 (J) hours after transduction with 
$5,10,20,35$ or $50 \mathrm{MOI}$ was determined by ELISA. LT $\alpha$ supernatant concentration was significantly increased following transduction with 35 and $50 \mathrm{MOI}$ LVLT $\alpha$ compared to control cells (medium only) and LVGFP ( $p=0.0422$ and 0.0059 respectively, Kruskal-Wallis with Dunn's post-test). (K) Transduction of passaged meningeal cells with lentiviral vectors following longer term culture. LT $\alpha$ supernatant concentration was significantly increased at 7 days post-transduction of meningeal cells with $2000 \mathrm{MOI}$ LVLT $\alpha$ compared to control or LVGFP. (L) A western blot image of meningeal cell lysates at 72 hours post-LVGFP or LVLT $\alpha$ transduction showed a $42 \mathrm{kDa}$ band corresponding to $\beta$-actin in all lanes and a $22 \mathrm{kDa}$ band corresponding to human LT $\alpha$ post LVLTA but not LVGFP transduction. Statistics - 1-way analysis of variance with Tukey post-test. 
Supplementary Table 1: Demographic and clinical characteristics of the post-mortem cases used for CSF analysis and immunohistochemistry

\begin{tabular}{|c|c|c|c|c|c|}
\hline Cases & Sex & $\begin{array}{l}\text { Age at } \\
\text { Death }\end{array}$ & Cause of death & PM interval (hrs) & Disease duration (yrs) \\
\hline \multicolumn{6}{|c|}{ Control cases } \\
\hline $\mathrm{C5}$ & $\mathrm{F}$ & 95 & Bronchopneumonia & 9 & - \\
\hline $\mathrm{C7}$ & $\mathrm{F}$ & 85 & Esophageal cancer & 9 & - \\
\hline C14 & $M$ & 64 & Cardiac failure & 18 & - \\
\hline $\mathrm{C} 28$ & $\mathrm{~F}$ & 60 & Ovarian cancer & 13 & - \\
\hline $\mathrm{C} 30$ & $M$ & 75 & Bronchopneumonia & 17 & - \\
\hline C32 & $M$ & 88 & Prostrate cancer & 12 & - \\
\hline C36 & $M$ & 68 & Cardiac failure & 30 & - \\
\hline C45 & $M$ & 77 & Cardiac failure & 22 & - \\
\hline C48 & M & 68 & Colon cancer & 10 & \\
\hline C50 & $M$ & 32 & Haemangiopericytoma, & 6 & - \\
\hline C51 & $M$ & 68 & Ischemic heart disease & 24 & - \\
\hline C54 & $M$ & 66 & Pancreatic Cancer & 16 & - \\
\hline PDC8 & $\mathrm{F}$ & 71 & Myocardial infarction & NA & - \\
\hline PDC22 & $M$ & 75 & Lung carcinoma & 10 & - \\
\hline PDC32 & $\mathrm{F}$ & 91 & Colon cancer & 12 & - \\
\hline PDC36 & $\mathrm{F}$ & 57 & Breast cancer & 15 & - \\
\hline \multicolumn{6}{|c|}{ MS cases - low meningeal inflammation } \\
\hline MS104 & M & 53 & Multiple sclerosis & 12 & 11 \\
\hline MS296 & M & 59 & Multiple sclerosis & 22 & 40 \\
\hline MS301 & $\mathrm{F}$ & 62 & Septicaemia, MS & 16 & 20 \\
\hline MS304 & M & 52 & Pulmonary embolism & 13 & 23 \\
\hline MS311 & $\mathrm{F}$ & 45 & Pneumonia & 22 & 17 \\
\hline MS318 & $\mathrm{F}$ & 59 & Multiple sclerosis & 13 & 34 \\
\hline MS326 & M & 62 & Prostate cancer, MS & 24 & 32 \\
\hline MS335 & $M$ & 62 & Renal failure & 22 & 38 \\
\hline
\end{tabular}




\begin{tabular}{|c|c|c|c|c|c|}
\hline MS347 & $\mathrm{M}$ & 50 & Pancreatic carcinoma & 13 & 28 \\
\hline MS364 & $\mathrm{F}$ & 56 & Bronchopneumonia & 14 & 34 \\
\hline MS376 & $\mathrm{F}$ & 58 & Multiple sclerosis & 19 & 21 \\
\hline MS422 & $\mathrm{M}$ & 58 & Bronchopneumonia & 25 & 13 \\
\hline MS444 & $\mathrm{M}$ & 49 & Renal failure & 18 & 20 \\
\hline MS461 & $\mathrm{M}$ & 43 & Bronchopneumonia & 13 & 21 \\
\hline MS485 & $\mathrm{F}$ & 57 & Bronchopneumonia & 24 & 29 \\
\hline \multicolumn{6}{|c|}{ MS cases - high meningeal inflammation } \\
\hline MS180 & $\mathrm{F}$ & 44 & Multiple sclerosis & 9 & 18 \\
\hline MS234 & $\mathrm{F}$ & 39 & Aspiration pneumonia & 15 & 15 \\
\hline MS289 & $\mathrm{M}$ & 45 & Multiple sclerosis & 9 & 18 \\
\hline MS317 & $F$ & 48 & Aspiration pneumonia & 21 & 30 \\
\hline MS330 & F & 59 & Bronchopneumonia & 21 & 40 \\
\hline MS352 & $\mathrm{M}$ & 43 & Bronchopneumonia & 26 & 19 \\
\hline MS356 & $\mathrm{F}$ & 45 & Multiple sclerosis & 10 & 16 \\
\hline MS371 & $\mathrm{M}$ & 40 & Bronchopneumonia & 27 & 17 \\
\hline MS377 & $\mathrm{F}$ & 50 & Aspiration pneumonia & 22 & 23 \\
\hline MS402 & $\mathrm{M}$ & 46 & Multiple sclerosis & 12 & 20 \\
\hline MS407 & $\mathrm{F}$ & 44 & Septicaemia & 22 & 19 \\
\hline MS408 & $\mathrm{M}$ & 39 & Pneumonia & 21 & 10 \\
\hline MS423 & $\mathrm{F}$ & 54 & Pneumonia & 11 & 30 \\
\hline MS426 & $\mathrm{F}$ & 48 & Pneumonia & 21 & 19 \\
\hline MS438 & $\mathrm{F}$ & 53 & Multiple sclerosis & 17 & 18 \\
\hline MS473 & $\mathrm{F}$ & 40 & Broncho pneumonia & 9 & 13 \\
\hline MS497 & $\mathrm{F}$ & 60 & Aspiration pneumonia & 26 & 29 \\
\hline MS513 & $\mathrm{M}$ & 51 & Multiple sclerosis & 17 & 18 \\
\hline MS517 & $\mathrm{F}$ & 48 & Pulmonary sepsis & 12 & 25 \\
\hline MS528 & $F$ & 45 & Multiple sclerosis & 17 & 25 \\
\hline
\end{tabular}


Details of the control cases, and $M S$ cases. $M=$ male; $F=$ female; $P M D=$ post-mortem delay; yrs = years; NA = information not available. Cause of death is the primary cause taken from the certificate of death.

Supplementary Table 2: Demographics and disease characteristics of the MS subjects

\begin{tabular}{|c|c|c|}
\hline \multirow[t]{2}{*}{ Group } & \multicolumn{2}{|c|}{ Naïve MS patients } \\
\hline & Low (13) & High (18) \\
\hline Age studied (mean $\pm S D$ ) & $38.4 \pm 11.3$ & $33.2 \pm 11.1$ \\
\hline Gender (f:m) & $8: 5$ & $13: 5$ \\
\hline Disease duration (mean $\pm S D$ years) & $4.1 \pm 2.4$ & $5.3 \pm 2.0$ \\
\hline EDSS at recruitment (range) & $1.5(1.0-3.5)$ & $2.0(1.0-4.0)$ \\
\hline CLs volume $\left(\mathrm{mm}^{3}\right)$ (range) & $\begin{array}{l}49 \pm 74 \\
(0-188)\end{array}$ & $\begin{array}{l}1713 \pm 663 \\
(877-2678)\end{array}$ \\
\hline CLs number (mean $\pm S D$ ) & $\begin{array}{l}0.3 \pm 0.5 \\
(0-1)\end{array}$ & $\begin{array}{l}16.8 \pm 6.4 \\
(10-29)\end{array}$ \\
\hline CTh (mm; range) & $\begin{array}{l}2.72 \pm 0.41 \\
(2.45-3.15)\end{array}$ & $\begin{array}{l}2.53 \pm 0.34 \\
(2.31-2.99)\end{array}$ \\
\hline T2WMLV ( $\mathrm{cm}^{3} ;$ range) & \begin{tabular}{|l}
$4.7 \pm 1.6$ \\
$(1.4-7.2)$
\end{tabular} & $\begin{array}{l}5.6 \pm 1.6 \\
(3.1-8.4)\end{array}$ \\
\hline
\end{tabular}

Abbreviations: EDSS: Expanded Disability Status Scale; GM: grey matter; CL: cortical lesion; CTh: cortical thickness; T2WMLV: T2 white matter lesion volume. Low and High refer to the numbers of cortical lesions on MRI. 
A

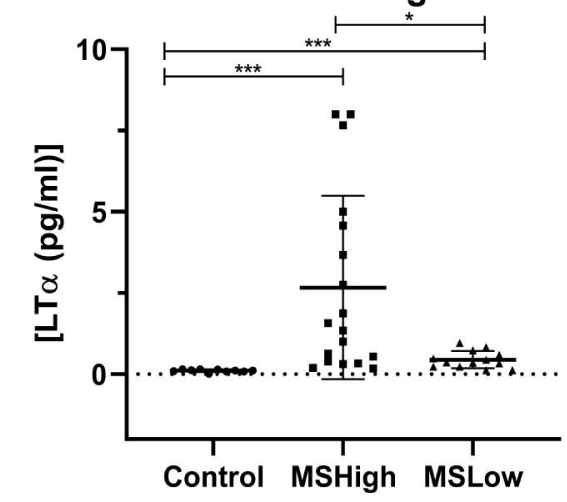

B Post-mortem SPMS CSF

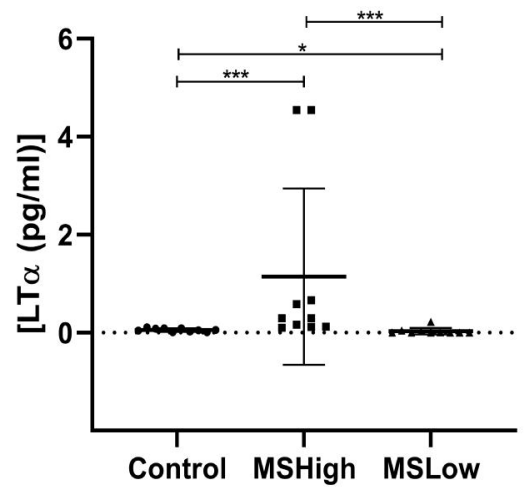

C Meninges - RNA

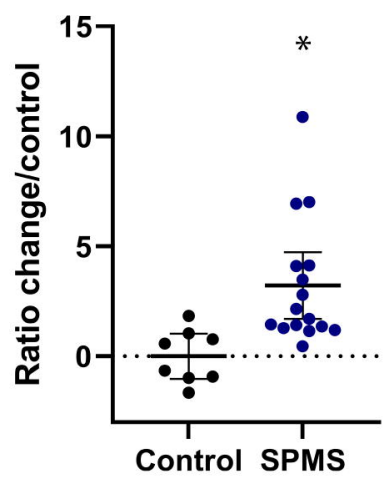

D Lymphotoxin- $\alpha$

F

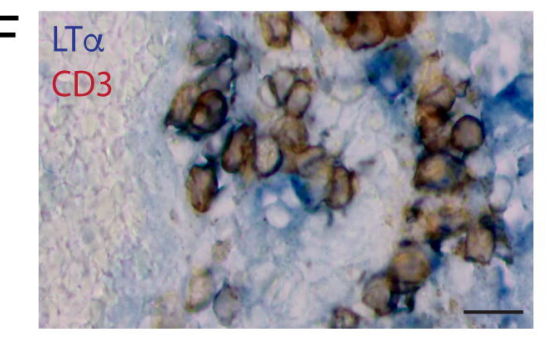

E Lymphotoxin-2\%/CD3

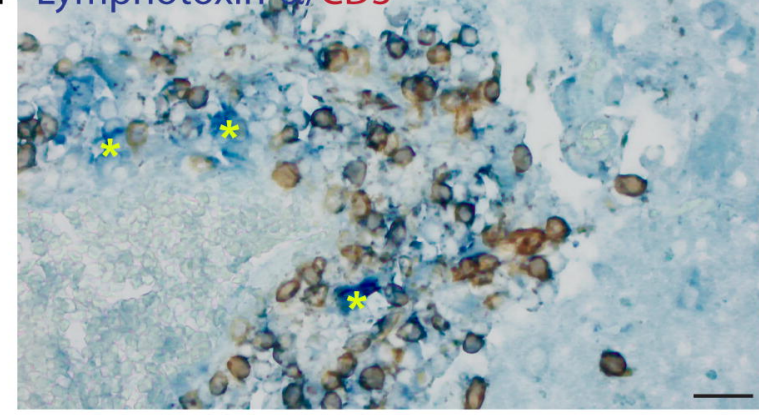

G

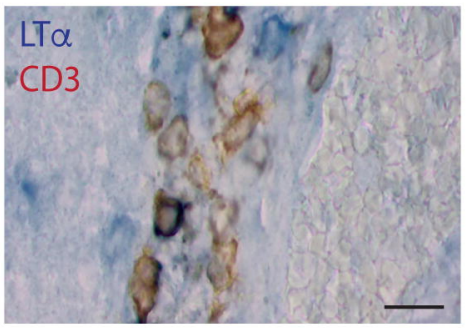

$\mathrm{H}_{\mathrm{L} T} \mathrm{TR}$

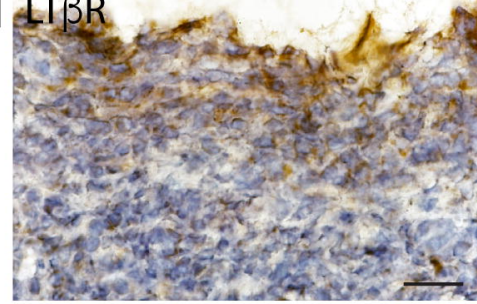




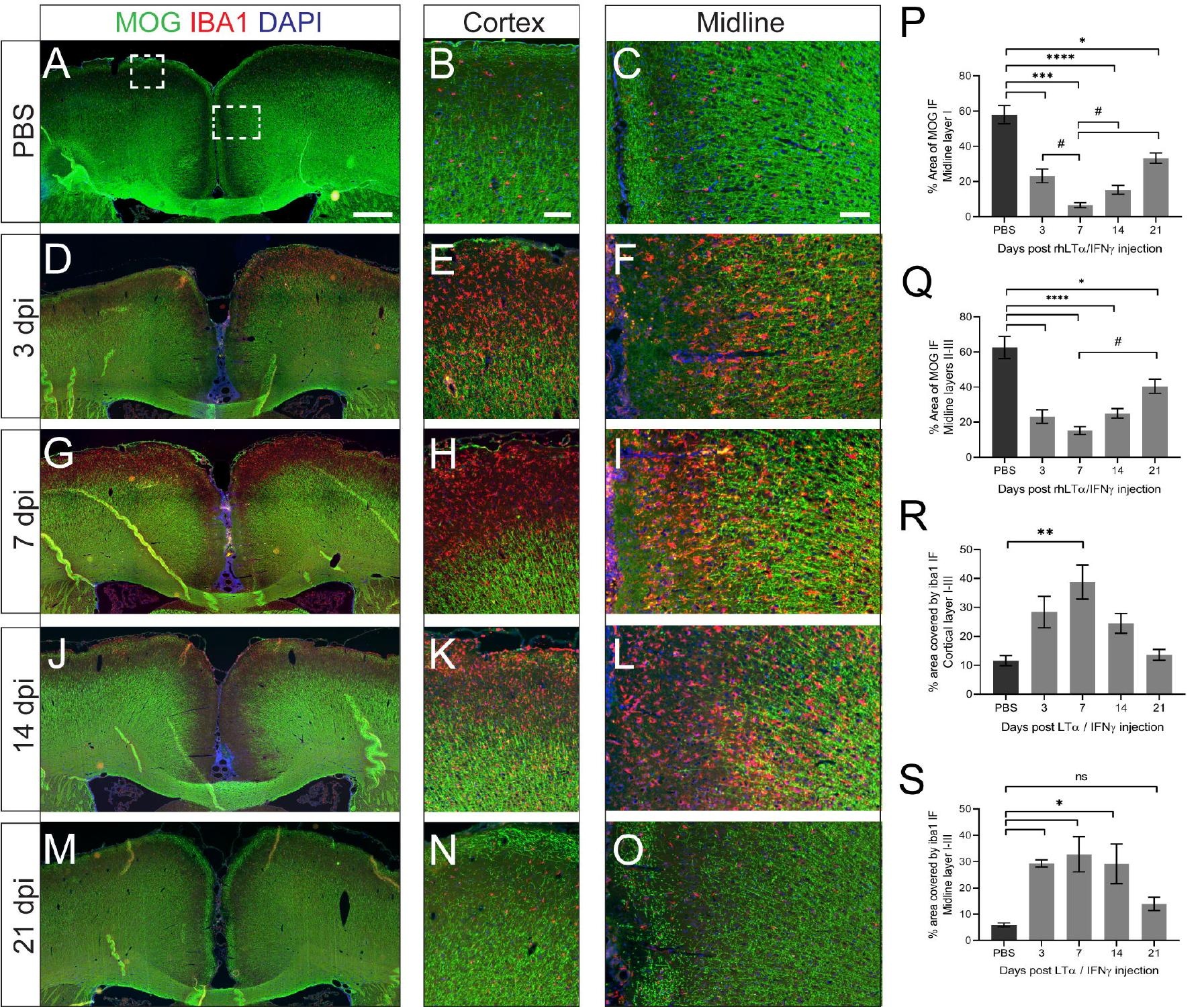


B MOG 28dpi

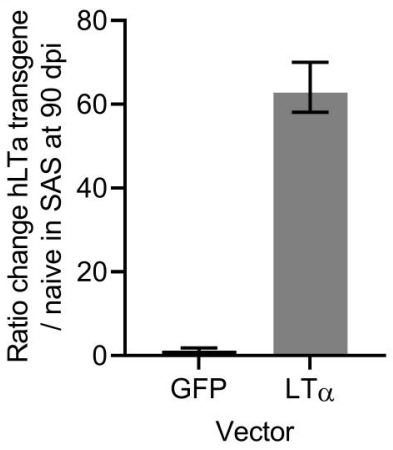

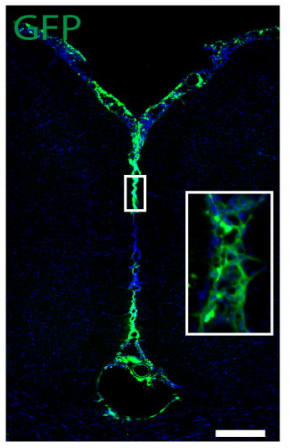

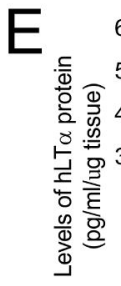

$\mathrm{D}$

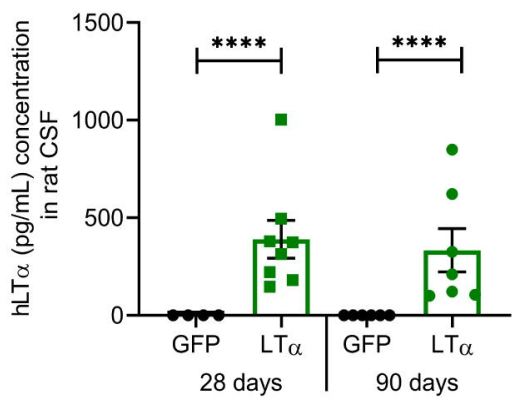

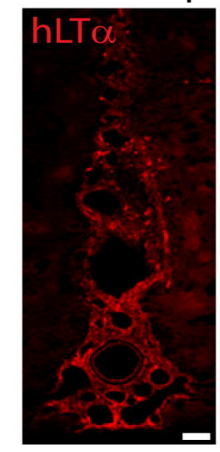

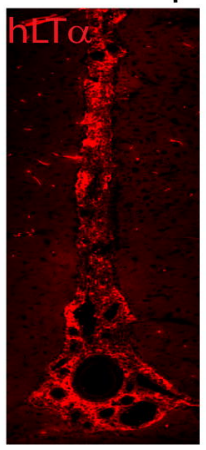

C MOG 28dpi MOG 90dpi

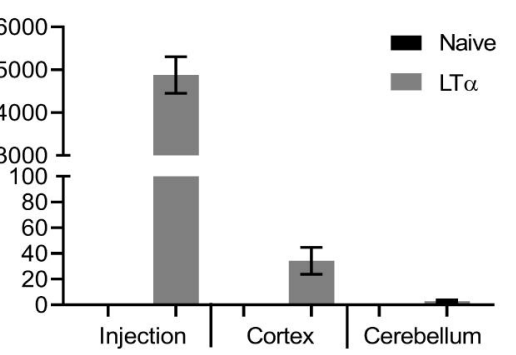

Rat brain lysate location 
$A$

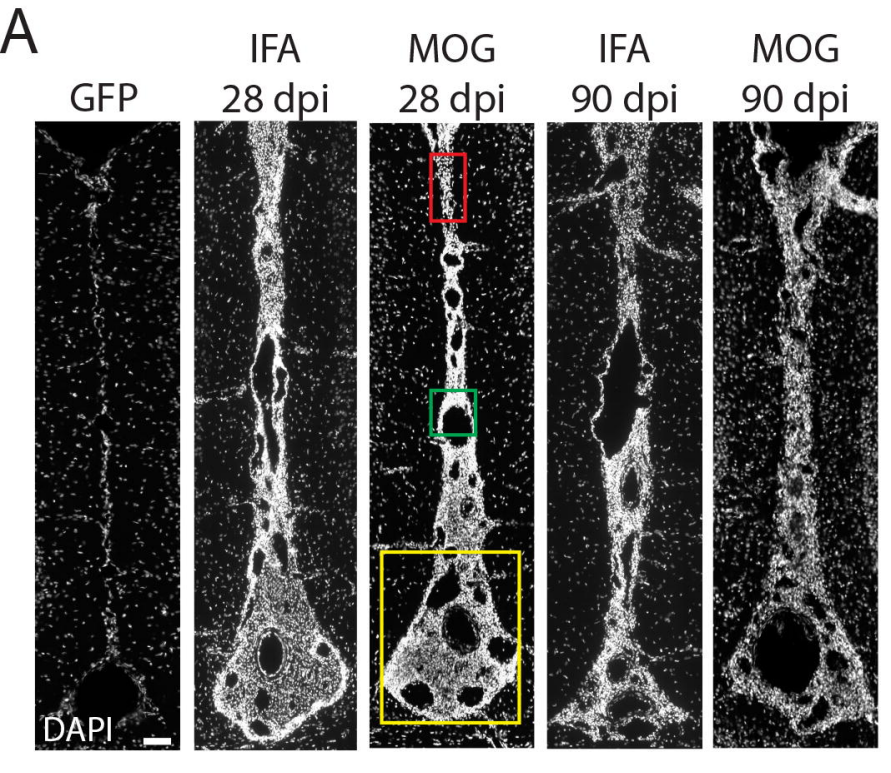

$\mathrm{E}$
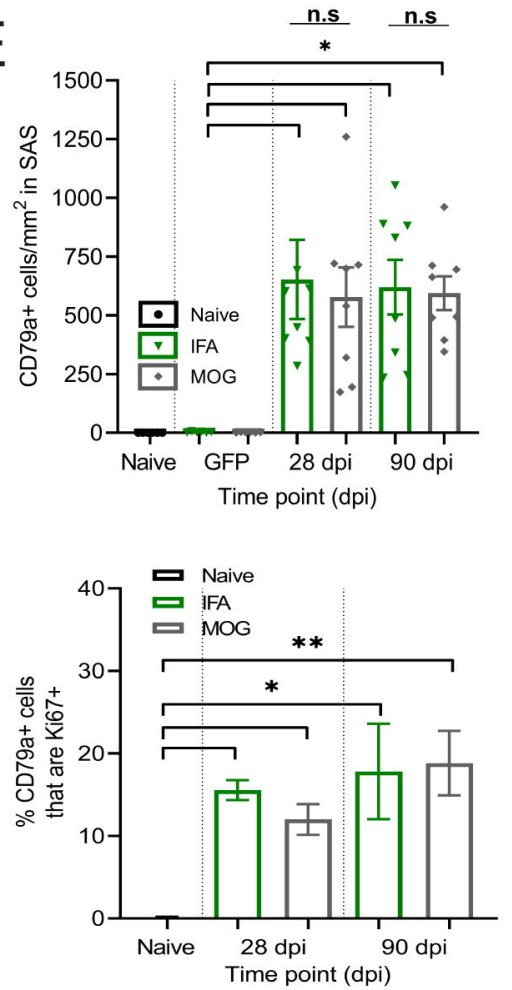

B

$\mathrm{CD} 4$

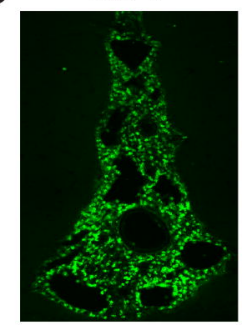

C

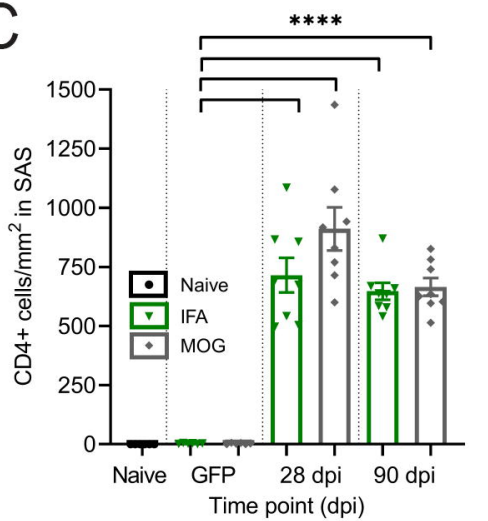

CD79a

Iba1

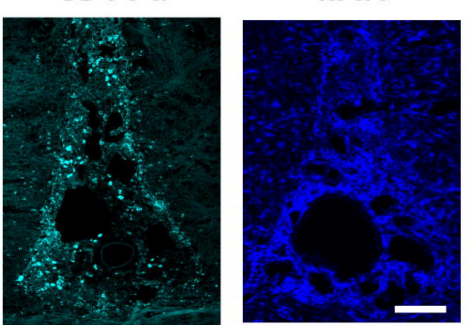

D

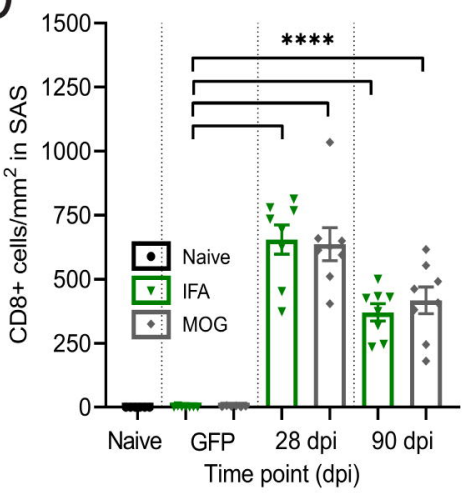

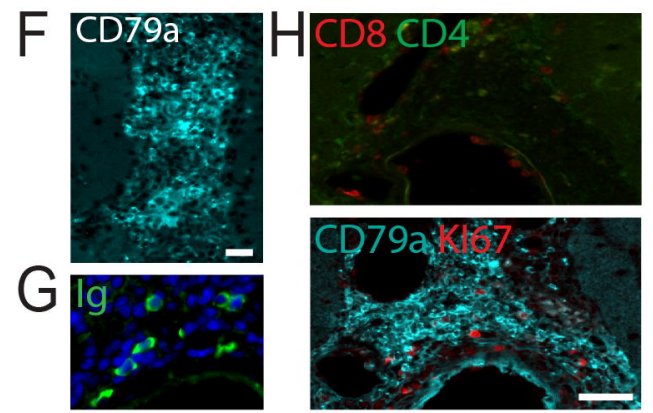

J

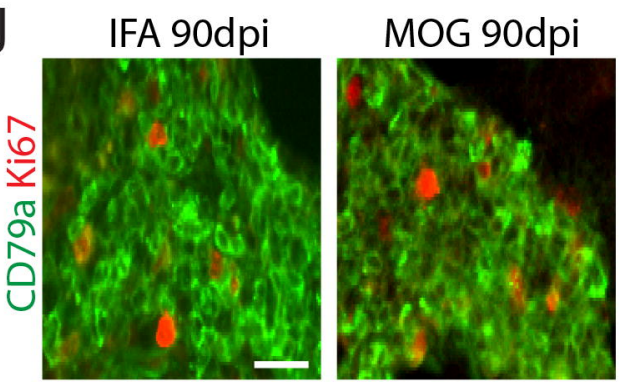

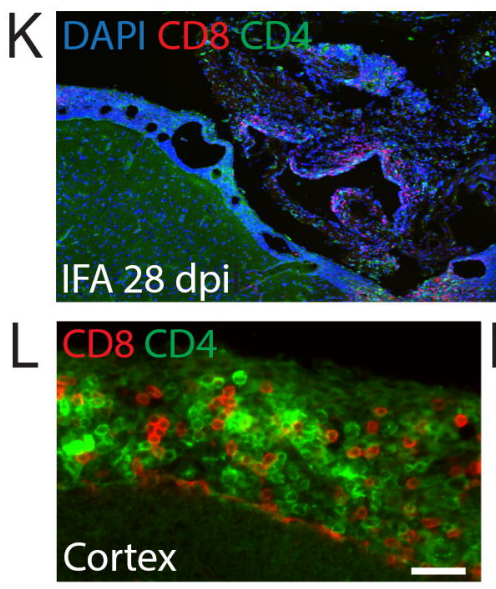
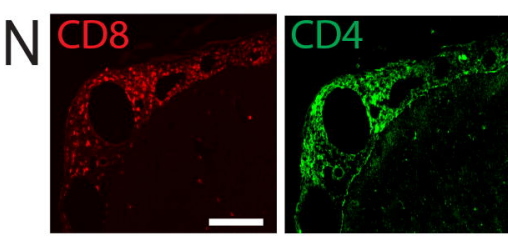

M CD79a

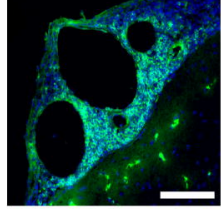

CD79a 


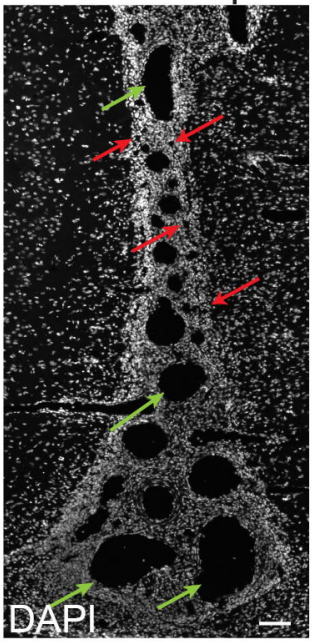

$90 \mathrm{dpi}$

B

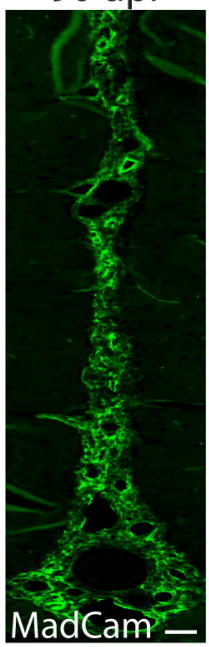

IFA

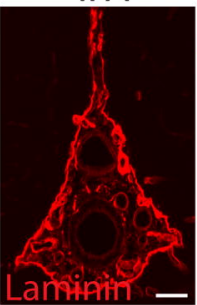

F Podoplanin

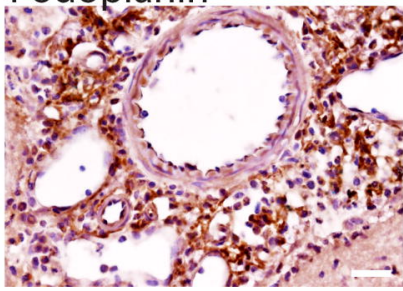

G
MOG

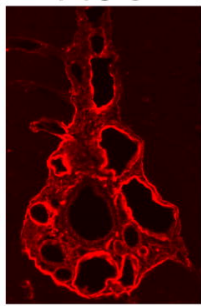

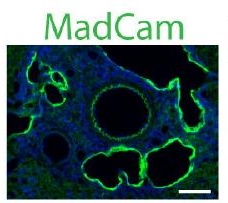
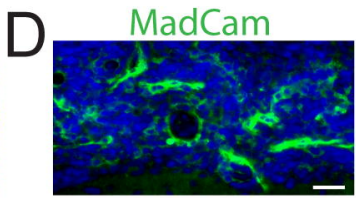

IFA

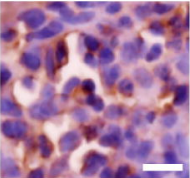

MOG

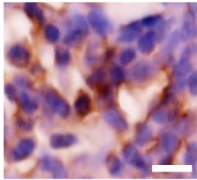


A $\quad \underset{\text { meninges }}{L T \alpha \text { treated primary }} \quad B$

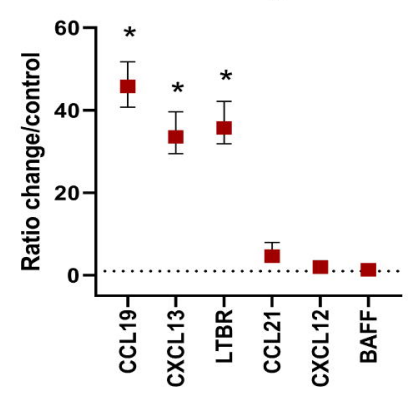

F Follicular dendritic cells

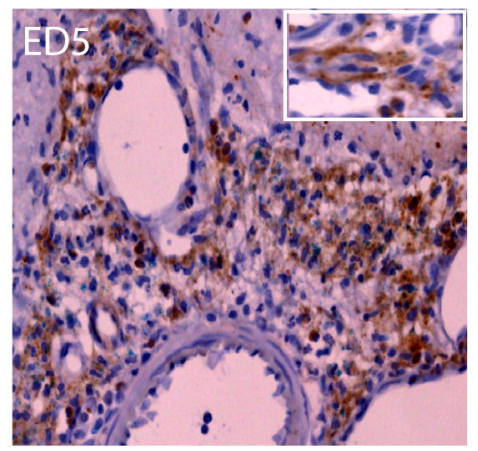

mRNA in meninges
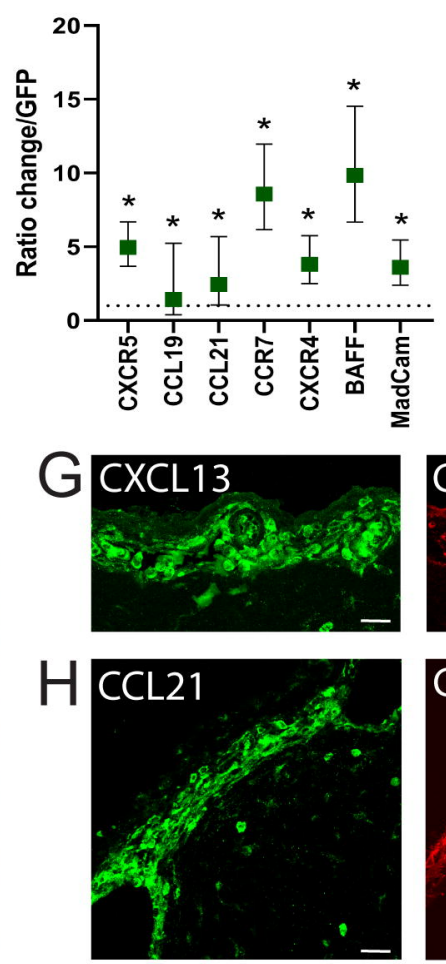

C

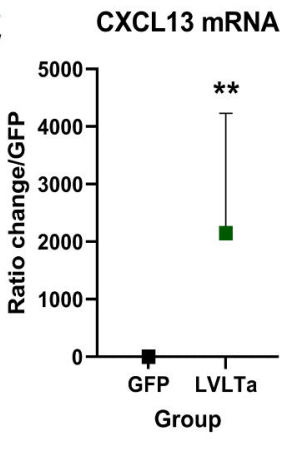

$D$ CXCL13

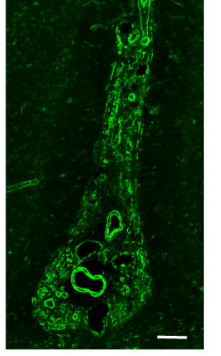

E CXCA13

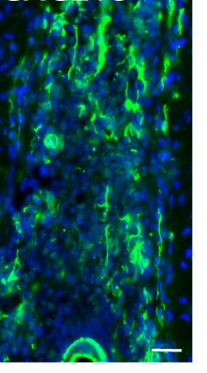

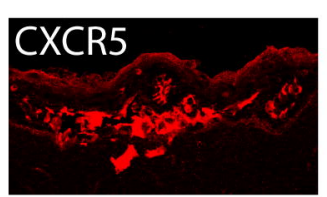

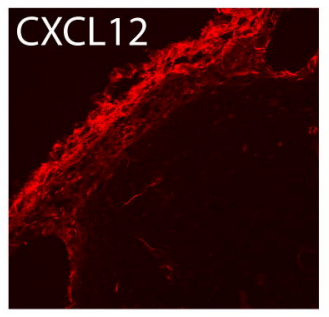

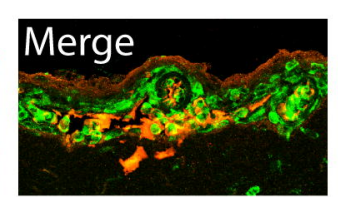

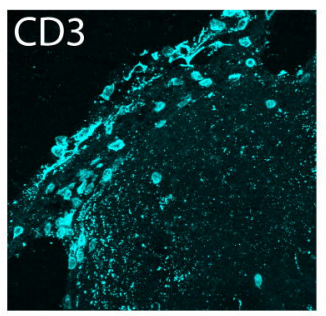

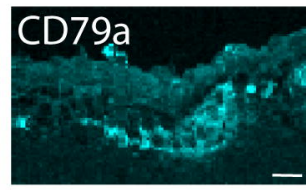

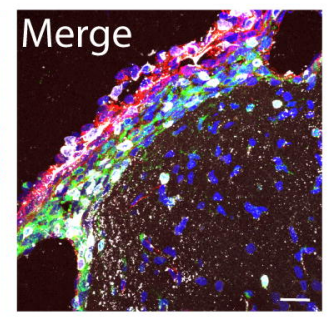

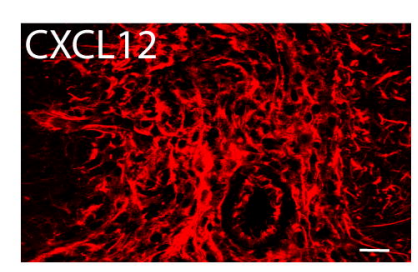

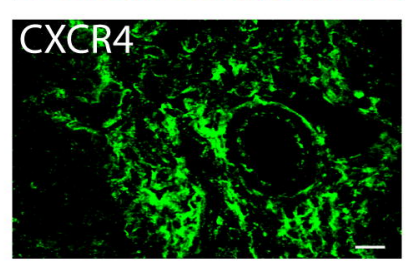

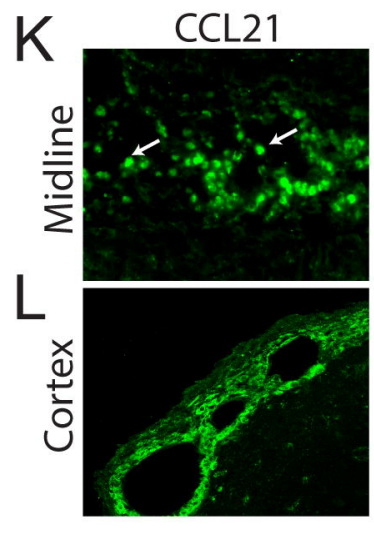

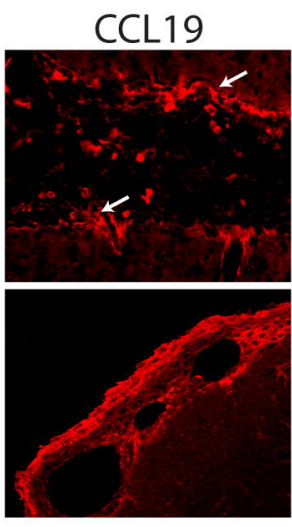

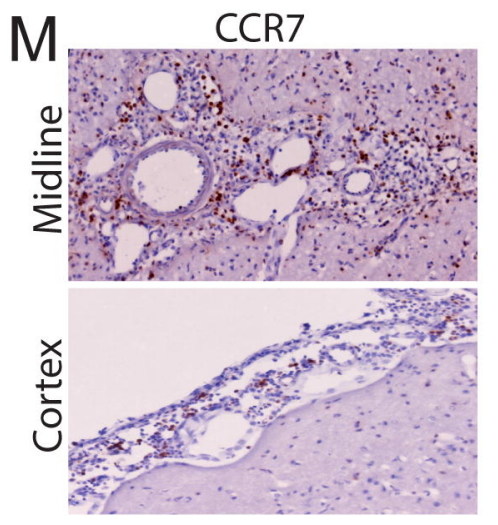


$A$

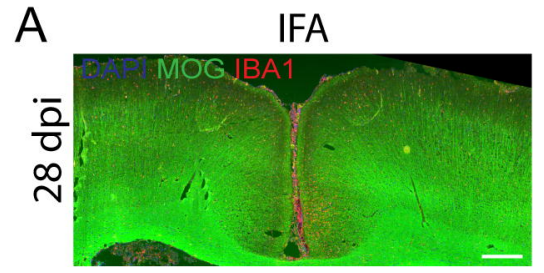

$B$
$\overline{ }$
응

C

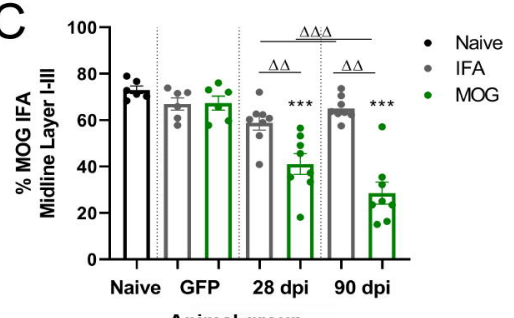

$\mathrm{H}$
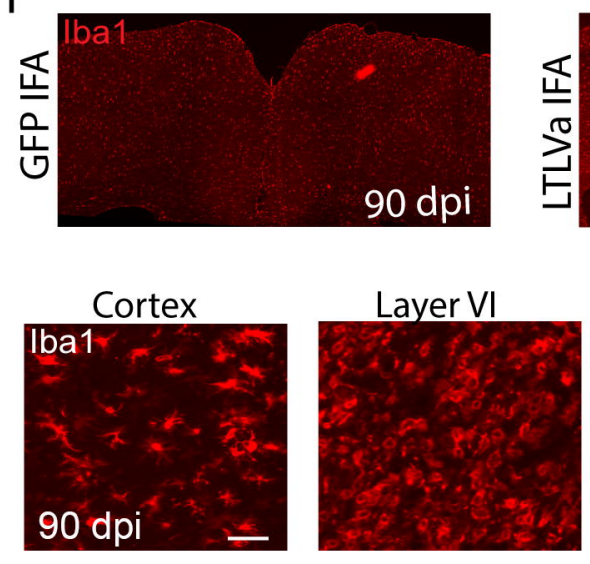

MOG
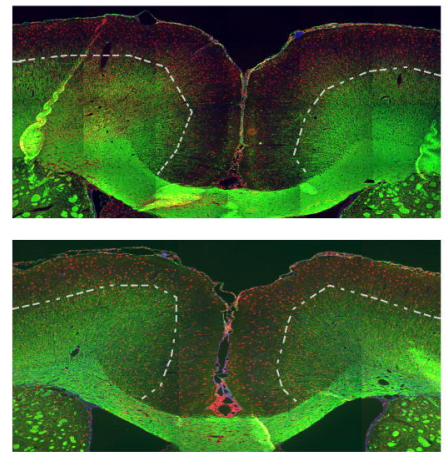

D

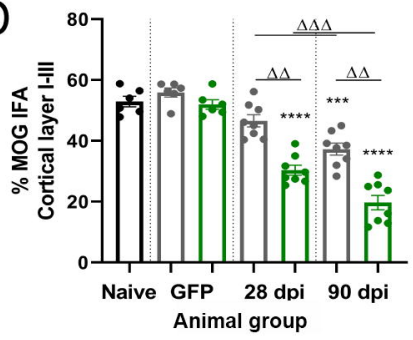

峜
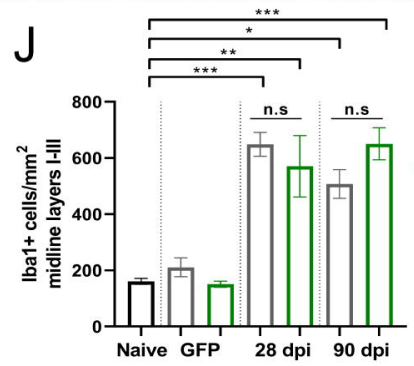

Animal group
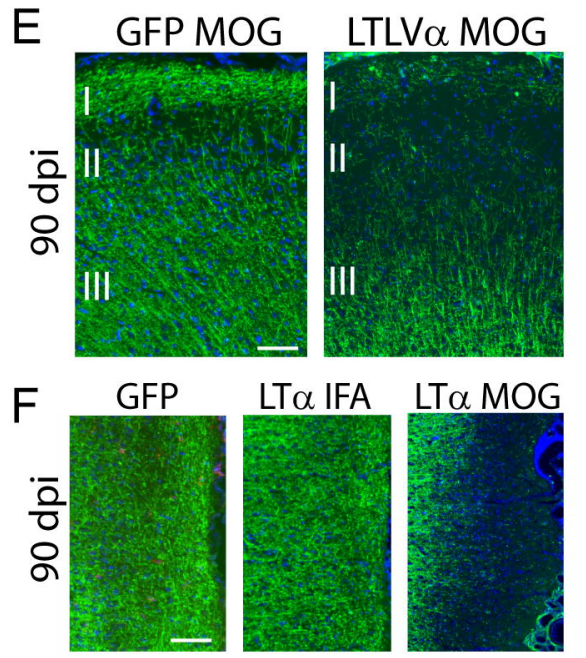

G
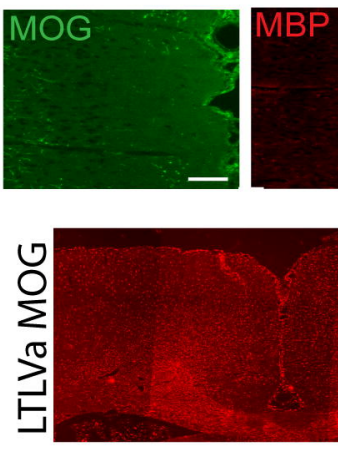

$\mathrm{K}$

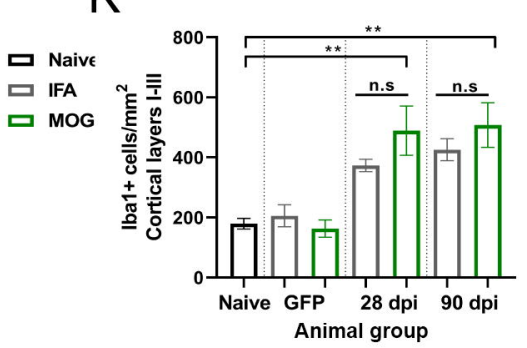


